\title{
Genomic and transcriptomic analyses reveal distinct biological functions for cold shock proteins (VpaCspA and VpaCspD) in Vibrio parahaemolyticus CHN25 during low-temperature survival
}

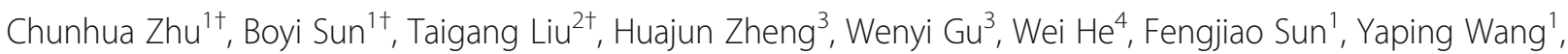
Meicheng Yang ${ }^{5}$, Weicheng Bei ${ }^{6}$, Xu Peng ${ }^{7}$, Qunxin She ${ }^{7}$, Lu Xie ${ }^{8^{*}}$ and Lanming Chen ${ }^{1^{*}}$

\begin{abstract}
Background: Vibrio parahaemolyticus causes serious seafood-borne gastroenteritis and death in humans. Raw seafood is often subjected to post-harvest processing and low-temperature storage. To date, very little information is available regarding the biological functions of cold shock proteins (CSPS) in the low-temperature survival of the bacterium. In this study, we determined the complete genome sequence of $V$. parahaemolyticus CHN25 (serotype: O5:KUT). The two main CSP-encoding genes (VpacspA and VpacspD) were deleted from the bacterial genome, and comparative transcriptomic analysis between the mutant and wild-type strains was performed to dissect the possible molecular mechanisms that underlie low-temperature adaptation by $V$. parahaemolyticus.

Results: The 5,443,401-bp V. parahaemolyticus CHN25 genome (45.2\% G + C) consisted of two circular chromosomes and three plasmids with 4,724 predicted protein-encoding genes. One dual-gene and two single-gene deletion mutants were generated for VpacspA and VpacspD by homologous recombination. The growth of the $\triangle V$ pacspA mutant was strongly inhibited at $10^{\circ} \mathrm{C}$, whereas the $V p a c s p D$ gene deletion strongly stimulated bacterial growth at this low temperature compared with the wild-type strain. The complementary phenotypes were observed in the reverse mutants ( $\triangle V$ pacspAcom, and $\triangle V$ pacspD-com). The transcriptome data revealed that $12.4 \%$ of the expressed genes in $V$. parahaemolyticus $\mathrm{CHN} 25$ were significantly altered in the $\triangle V$ pacspA mutant when it was grown at $10^{\circ} \mathrm{C}$. These included genes that were involved in amino acid degradation, secretion systems, sulphur metabolism and glycerophospholipid metabolism along with ATP-binding cassette transporters. However, a low temperature elicited significant expression changes for $10.0 \%$ of the genes in the $\Delta V$ pacspD mutant, including those involved in the phosphotransferase system and in the metabolism of nitrogen and amino acids. The major metabolic pathways that were altered by the dual-gene deletion mutant $(\triangle V$ pacspAD) radically differed from those that were altered by single-gene mutants. Comparison of the transcriptome profiles further revealed numerous differentially expressed genes that were shared among the three mutants and regulators that were specifically, coordinately or antagonistically modulated by VpaCspA and VpaCspD. Our data also revealed several possible molecular coping strategies for low-temperature adaptation by the bacterium. (Continued on next page)
\end{abstract}

\footnotetext{
* Correspondence: xielu@scbit.org; Imchen@shou.edu.cn

${ }^{\dagger}$ Equal contributors

${ }^{8}$ Shanghai Center for Bioinformation Technology, 1278 Keyuan Road,

Shanghai 201203, People's Republic of China

'Key Laboratory of Quality and Safety Risk Assessment for Aquatic Products

on Storage and Preservation (Shanghai), China Ministry of Agriculture;

College of Food Science and Technology, Shanghai Ocean University, 999

Hu Cheng Huan Road, Shanghai 201306, People's Republic of China

Full list of author information is available at the end of the article
} 
(Continued from previous page)

Conclusions: This study is the first to describe the complete genome sequence of $\mathrm{V}$. parahaemolyticus (serotype: O5:KUT). The gene deletions, complementary insertions, and comparative transcriptomics demonstrate that VpaCspA is a primary CSP in the bacterium, while VpaCspD functions as a growth inhibitor at $10{ }^{\circ} \mathrm{C}$. These results have improved our understanding of the genetic basis for low-temperature survival by the most common seafood-borne pathogen worldwide.

Keywords: Vibrio parahaemolyticus, Complete genome sequence, Cold shock protein, Gene deletion, Transcriptome, Low-temperature adaptation

\section{Background}

Vibrio parahaemolyticus naturally occurs in marine, estuarine and aquaculture environments worldwide and causes serious seafood-borne gastroenteritis and death in humans, particularly when raw, undercooked or mishandled seafood is consumed [1, 2]. V. parahaemolyticus was initially identified in 1950 in Osaka, Japan, where an outbreak of acute gastroenteritis that was caused by the consumption of semidried juvenile sardines sickened 272 people and killed 20 [3]. To date, over eighty $V$. parahaemolyticus serotypes have been described based on the somatic $(\mathrm{O})$ and capsular (K) antigens [1]. Of these serotypes, complete genome sequences have been published for three $V$. parahaemolyticus strains-RIMD2210633 (serotype: O3:K6) [4], BB22OP (serotype: O4:K8) [5] and UCM-V493 (serotype: O2:K28) strains [6]. Additionally, two complete and multiple draft genome sequences for the V. parahaemolyticus strains are available in the GenBank database (http:// www.ncbi.nlm.nih.gov/genome/) and online (http:// www.genomesonline.org) [7-9].

$V$. parahaemolyticus is a gram-negative bacterium that is frequently isolated from raw seafood [2]. Seafood is often subjected to post-harvest processing and lowtemperature storage, during which the bacterium is challenged to survive under detrimental cold conditions. Previous studies have indicated that the temperature decrease elicits complex cold shock responses in foodrelated bacteria (e.g., lactic acid bacteria, food spoilage bacteria and food-borne pathogens), such as the regulation of uptake or synthesis of compatible solutes, DNA supercoiling modifications, membrane fluidity maintenance, and cold shock protein (CSP) production (for a review, see [10]).

CSPs comprise a group of low-molecular-weight proteins of approximately $7 \mathrm{kDa}$. CSP families that contain between two and nine members have been identified in food-related bacteria and several food-borne pathogens, including Escherichia coli, Listeria monocytogenes, Staphylococcus aureus, Salmonella typhimurium and Pseudomonas fragi [11]. In E. coli, the CSP family contains nine members (A-I), of which CspA is a well characterised RNA chaperone that reduces low temperature-associated increases in RNA secondary folding [10]. Although CSPs share a high degree of sequence similarity $(>45 \%)$ with two conserved RNA-binding motifs, it is surprising that not all CSP members are cold-inducible, which implies that they may function in different cellular processes [11]. CspD in E. coli reportedly plays a negative regulatory role in chromosomal replication in nutrient-depleted cells [12]. Recent studies have indicated that the MqsR/MqsA toxin/ antitoxin pair directly regulates $\mathrm{CspD}$, which may be involved in toxicity and biofilm formation in E. coli [13].

Despite its significance in human health and in the aquaculture industry, the molecular mechanisms that underlie the low-temperature survival of $V$. parahaemolyticus remain largely unknown. Previous studies have revealed three E. coli CSP homologues in V. parahaemolyticus, including CspA, CspD and the cold shock DNA-binding domain-containing protein [14]. The $\operatorname{csp} A$ gene was upregulated at the transcriptional level by over 30 -fold after V. parahaemolyticus was treated for $60 \mathrm{~min}$ at $10{ }^{\circ} \mathrm{C}$, a temperature below which bacterial growth was arrested [14]. However, the genes that encoded the other two homologues were undetectable by DNA microarray and realtime reverse transcription PCR (qRT-PCR) [14], which suggested that CspA could be a major CSP in V. parahaemolyticus during low-temperature growth. This study is the first to sequence, assemble and annotate the complete genome of $V$. parahaemolyticus CHN25 (serotype: O5:KUT), which has recently been isolated and characterised [15-17]. We constructed one dual-gene and two single-gene deletion mutants of the two main $V$. parahemolyticus CHN25 CSPs (designated as VpaCspA and $V p a C s p D)$ and determined the global-level gene expression profiles of the mutant and wild-type strains by Illumina RNA-Sequencing. These data will refine our grasp of the molecular mechanisms that underlie the lowtemperature adaptation of the most common seafoodborne pathogen worldwide.

\section{Results and discussion}

\section{Genomic features of $V$. parahaemolyticus CHN25}

The complete genome sequence of $V$. parahaemolyticus CHN25 was determined by 454-pyrosequencing (see Methods). It consisted of two circular chromosomes that 
contained 3,416,467 bp and 1,843,316 bp (see Additional file 1: Figure S1). The genome also contained three plasmids (92,495 bp, 83,481 bp and 7,642 bp), all of which were absent from the other known $V$. parahaemolyticus genomes (see Additional file 2: Figure S2). The complete $V$. parahaemolyticus $\mathrm{CHN} 25$ genome contained 5,443,401 bp with a $45.2 \% \mathrm{G}+\mathrm{C}$ content; 4,724 protein-encoding genes were predicted, of which approximately $34.8 \%$ encoded hypothetical proteins with unknown functions in public databases. Additionally, 9 rRNA operons and 55 ribosomal protein-encoding genes, 107 tRNA genes, and 30 pseudogenes were identified and annotated.

In marked contrast to the other known $V$. parahaemolyticus genomes, an integrative and conjugative element (ICEVpaChn1) was identified in the CHN25 genome. The 89.9-kb element (VpaChn25_2302 to Chn25_2378) contained sulfamethoxazole and streptomycin resistance genes. Mating assays demonstrated the active self-transmissibility of the antibiotic resistance from $V$. parahaemolyticus CHN25 to E. coli MG1655 [15]. Five prophage gene clusters that ranged from 6.5 to $36.6 \mathrm{~kb}$ were identified in the CHN25 genome, and they displayed high degrees of sequence identity with Vibrio phage martha 12B12 (GenBank accession no. HQ316581), Vibrio phage VPUSM 8 (GenBank accession no. KF361475), Vibrio phage henriette 12B8 (GenBank accession no. HQ316582), and Vibrio phage N4 [18]. Additionally, five insertion sequences (ISs) were detected in the genome, including ISShfr9 (Tn3), ISVal1, ISVpa3 (IS5) and ISVsa3 (IS91); the latter existed as two copies in the genome, which suggested that it was probably active. We concluded that the V. parahaemolyticus $\mathrm{CHN} 25$ genome has undergone major rearrangements due to its mobile genetic elements.

Consistent with the other $V$. parahaemolyticus genomes, most of the genes that encoded enzymes for the predicted central metabolic pathways were present in the CHN25 strain, including those required for glycolysis, oxidative phosphorylation and tricarboxylic acid cycle (TCA). Additionally, the CHN25 genome also contained genes for three restriction and modification (R-M) systems (types I, II and IV) and four DNA repair systems (base excision repair, nucleotide excision repair, mismatch repair and homologous recombination), most of which were present in several other $V$. parahaemolyticus strains. The high frequency of the horizontal gene transfer in the CHN25 strain (i.e., ICEVpaChn1) may have led the bacteria to hijack the R-M and DNA repair mechanisms to generate genetic diversity without losing genomic stability [19].

\section{Construction of the $\triangle V p a c s p A, \triangle V p a c s p D$ and $\triangle V p a c s p A D$ mutants of $V$. parahaemolyticus CHN25}

To investigate the low-temperature adaptation that was mediated by the predicted CSPs in $V$. parahaemolyticus CHN25, we constructed a deletion mutant of the VpacspA gene. The upstream and downstream sequences (approximately $0.5 \mathrm{~kb}$ ) that flanked the VpacspA gene were obtained by PCR and cloned into a suicide vector, pDS132, to yield the recombinant vector, pDS132 $+\triangle$ Vpacsp $A$. The inserted 1,041-bp sequence was confirmed by DNA sequencing (data not shown). The recombinant vector was transformed into $E$. coli $\beta 2155$, and the chloramphenicol-resistant transformant was obtained and conjugated with $V$. parahaemolyticus CHN25. Positive exconjugants were obtained using the two-step allelic exchange method (see the Methods section) and validated by PCR. DNA sequencing of the PCR product further confirmed the in-frame deletion of the 213-bp sequence of the VpacspA gene from the $V$. parahaemolyticus CHN25 genome (data not shown).

Similarly, the VpacspD gene that encoded a cold shock-like protein was deleted from the bacterial genome using the aforementioned method. The $\Delta$ VpacspD mutant with a 219-bp in-frame deletion was confirmed by DNA sequencing (data not shown). Furthermore, the VpacspD gene was also successfully deleted from the $\triangle$ VpacspA mutant, yielding a dual-gene deletion mutant of $\triangle V$ pacspAD (data not shown). The genome-level transcriptome data provided direct evidence of the successful construction of the three mutants, in which expression of the corresponding VpacspA or VpacspD genes was undetectable (see below).

\section{Survival of the $\triangle V p a c s p A, \triangle V p a c s p D$ and $\triangle V p a c s p A D$ mutants at $10^{\circ} \mathrm{C}$}

To gain insights into the possible effects of the CSPassociated gene deletions on $V$. parahaemolyticus CHN25 low-temperature survival, we determined growth curves for the $\triangle V p a c s p A, \triangle V p a c s p D$ and $\triangle V p a c s p A D$ mutants, which were grown in LB broth $(3 \% \mathrm{NaCl}, \mathrm{pH} 8.5)$ at $37^{\circ} \mathrm{C}$ or $10^{\circ} \mathrm{C}$. As shown in Fig. 1 (A), no apparent differences in growth were observed between the mutant and wildtype strains at $37{ }^{\circ} \mathrm{C}$, which was an optimal growth temperature. However, the $\triangle$ VpacspA mutant showed a longer lag phase $(>30 \mathrm{~h})$ and grew more slowly compared with the wild-type strain at $10{ }^{\circ} \mathrm{C}$ (Fig. 1b), demonstrating that VpaCspA was a crucial CSP in V. parahaemolyticus CHN25 low-temperature survival. Although $V p a C s p D$ was identified as one of the three homologues of the E. coli CSPs [14], the VpacspD gene deletion unexpectedly stimulated mutant growth at $10{ }^{\circ} \mathrm{C}$ in our study, which was notably faster than the wild-type strain (Fig. 1b), indicating that $\mathrm{VpaCspD}$ likely functioned as a lowtemperature bacterial growth inhibitor. A BLAST analysis revealed that the $\mathrm{VpaCspD}$ sequence shared a $70 \%$ amino acid identity with CspD in $E$. coli $(E c C s p D)$ (Fig. 2), which has been proposed to function as a novel inhibitor of DNA replication in nutrient-depleted cells [12]. Unlike $E c C s p D$, its null mutant grew well over a 15 to $42{ }^{\circ} \mathrm{C}$ 

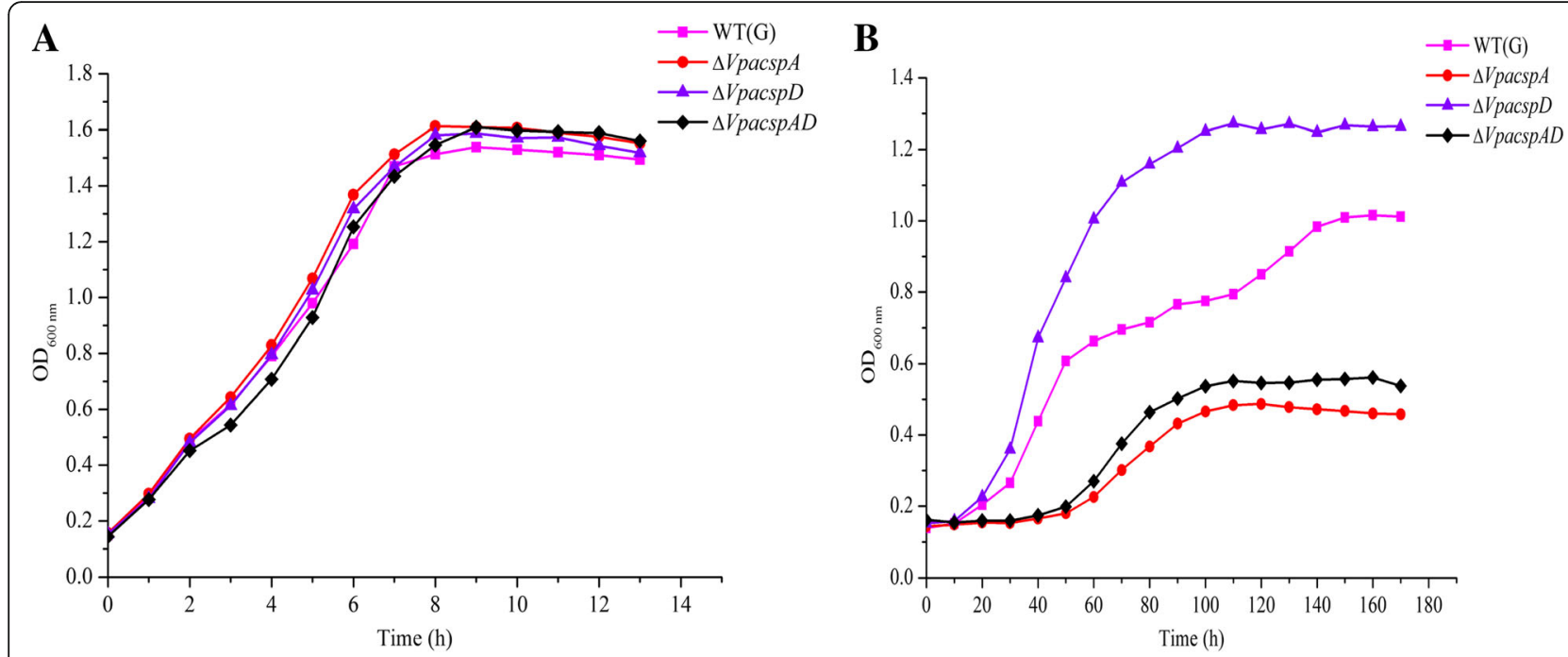

Fig. 1 Growth of $V$. parahaemolyticus CHN25 and the $\triangle V$ pacspA, $\triangle V$ pacspD and $\triangle V$ pacspAD mutants in $L B$ broth $(3 \% \mathrm{NaCl}, \mathrm{pH} 8.5)$ at $37^{\circ} \mathrm{C}(\mathbf{a})$ and $10{ }^{\circ} \mathrm{C}(\mathbf{b})$

temperature range with no detectable morphological changes. Our data indicated that VpacspD also functioned as a low-temperature induced-CSP (see below). Because only three CSP-associated genes were identified in $V$. parahaemolyticus and because $\mathrm{VpaCspD}$ only displayed a $48 \%$ amino acid sequence identity with $\mathrm{VpaCspA}$ (Fig. 2), $V p a C s p D$ may have evolved to gain different biological functions. Interestingly, the $\triangle V$ pacsp $A D$ mutant also grew poorly at low temperature compared to the wild-type strain (Fig. 1b), indicating that the phenotype of the VpacspA gene deletion dominated that of the VpacspD gene (see below).

\section{Construction of the reverse mutants $\triangle V$ pacspA-com and} $\triangle V$ pacspD-com and complementary phenotypes at $10^{\circ} \mathrm{C}$ The $\operatorname{csp} A$ gene was amplified from the genomic DNA of $V$. parahaemolyticus CHN25 by $\mathrm{PCR}$, and cloned into the expression vector pMMB207, which yielded the recombinant vector pMMB207-VpacspA. The inserted 213-bp sequence was confirmed by DNA sequencing (data not shown). This recombinant vector was then electrotransformed into the $\triangle V p a c s p A$ mutant competent cells, and generated the reverse mutant $\triangle V$ pacspA-com (see the Methods section). Similarly, the recombinant vector pMMB207-VpacspD carrying the 240-bp cspD gene was also constructed, and electrotransformed into the $\triangle V$ V a cspD mutant, yielding the reverse mutant $\triangle$ VpacspD-com. The growth curves for the reverse mutants $\triangle$ Vpacsp $A$-com and $\triangle$ Vpacs $p D$-com were also determined, which were incubated in LB broth $(3 \% \mathrm{NaCl}, 5 \mu \mathrm{g} / \mathrm{mL}$ chloramphenicol, $\mathrm{pH}$ 8.5) at $37{ }^{\circ} \mathrm{C}$ or $10{ }^{\circ} \mathrm{C}$ (Fig. 3). Consistent with the results in Fig. 1a, no obvious difference in growth at $37{ }^{\circ} \mathrm{C}$ was observed among the wild type, the mutants $\triangle V p a c s p A$ and $\triangle V$ pacspD, and the reverse mutants $\triangle V p a c s p A$-com and $\triangle$ VpacspD-com (Fig. 3a). However, at $10{ }^{\circ} \mathrm{C}$, the reverse mutants displayed similar growth phenotype as the wild type (Fig. 3b), demonstrating that the distinct phenotypes of the mutants $\triangle V$ pacsp $A$ and $\triangle V p a c s p D$ were indeed resulted from the VpacspA and VpacspD gene deletions in V. parahaemolyticus CHN25.

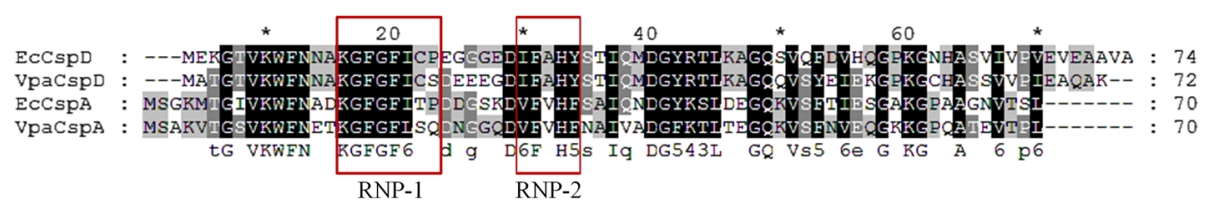

Fig. 2 A multi-sequence alignment of the CSPs from V. parahaemolyticus CHN25 and E. coli. The numbers above the alignments indicate the relative positions of the entirely aligned sequences. Identical and conserved ( $>50 \%$ of the sequences) amino acid residues are highlighted in black and grey, respectively; the consensus sequence is shown below the alignment. The RNA-binding motifs (RNP-1 and RNP-2) are boxed. The EcCspA and EcCspD sequences were derived from E. coli JM83 (Yamanaka et al. [12]), while the VpaCspA (VpaChn25A_0413) and VpaCspD (VpaChn25_1036) sequences were obtained from $V$. parahaemolyticus CHN25 in this study 

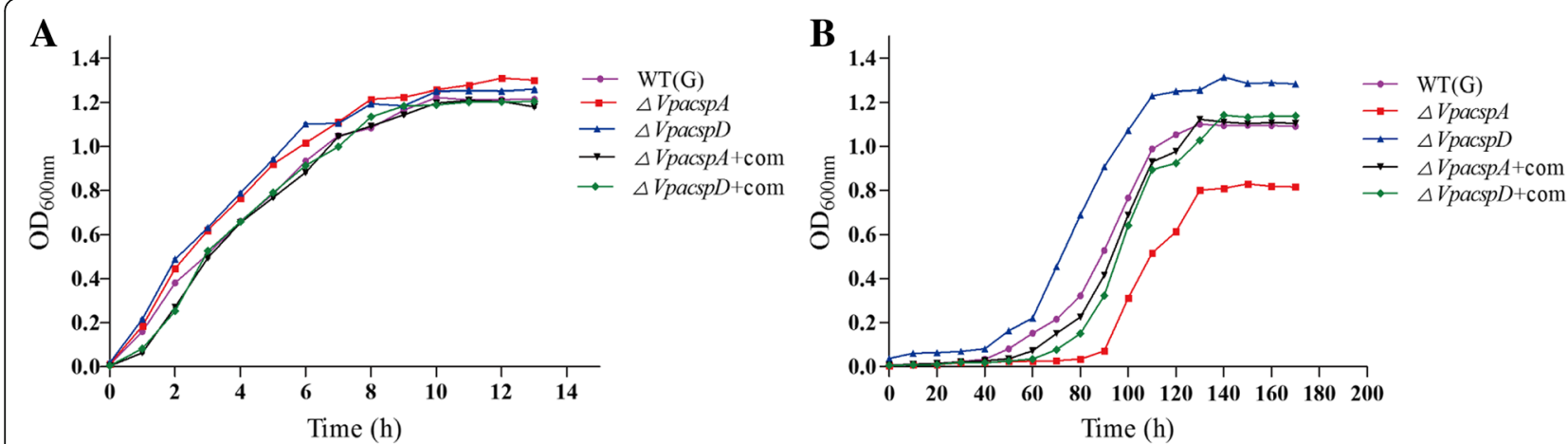

Fig. 3 Growth of $V$. parahaemolyticus CHN25, the mutants ( $\Delta V p a c s p A, \Delta V p a c s p D$ ), and the reverse mutants ( $\Delta V p a c s p A-c o m, \Delta V p a c s p D-c o m)$ at $37{ }^{\circ} \mathrm{C}(\mathbf{a})$ and $10{ }^{\circ} \mathrm{C}(\mathbf{b})$. The wild type and the mutants were incubated in $\mathrm{LB}$ broth $(3 \% \mathrm{NaCl}, \mathrm{pH} 8.5)$, and the reverse mutants in the $\mathrm{LB}$ supplemented with $5 \mu \mathrm{g} / \mathrm{mL}$ chloramphenicol

Transcriptome profiles for the $\triangle V p a c s p A, \triangle V p a c s p D$ and $\triangle$ VpacspAD mutants at $10^{\circ} \mathrm{C}$

To further investigate the $V p a C s p A$ - and $V p a C s p D-m e d i-$ ated low-temperature survival of $V$. parahaemolyticus $\mathrm{CHN} 25$, we determined global-level gene expression profiles for the $\triangle V p a c s p A, \triangle V p a c s p D$ and $\triangle V p a c s p A D$ mutants that were grown at $10{ }^{\circ} \mathrm{C}$, where distinct growth phenotypes were evident. Based on the complete genome sequence of $V$. parahaemolyticus $\mathrm{CHN} 25$, this analysis revealed numerous differentially expressed genes (DEGs) in the mutants, indicating that VpaCspA and $V p a C s p D$ likely functioned as master or global regulators in lowtemperature bacterial growth. Five hundred seventy-two genes were significantly altered in the $\Delta V p a c s p A$ mutant compared with the wild-type strain; these genes represented approximately $12.4 \%$ of the expressed genes in $V$. parahaemolyticus $\mathrm{CHN} 25$. Of these, 263 genes showed higher transcriptional levels (fold change $\geq 2.0$ ), while 309 genes were down-regulated (fold change $\leq 0.5$ ). The altered genes in the $\Delta V$ pacsp $A$ mutant were grouped into eighty-three gene functional catalogues that were identified in the Kyoto Encyclopaedia of Genes and Genomes (KEGG) database (data not shown). The VpacspD gene deletion elicited $10 \%$ of the differentially expressed genes in the bacterium, including 242 upregulated and 219 down-regulated genes that were grouped into seventy-six gene functional catalogues (data not shown). Additionally, the expression of 352 and 289 genes was up- and down-regulated, respectively, in the dual-gene deletion mutant $(\triangle V$ pacsp $A D)$, which accounted for $13.9 \%$ of the expressed genes; they were grouped into seventy-four gene functional catalogues (data not shown). A complete list of the DEGs for the three mutants is available in the Gene Expression Omnibus database (http:// www.ncbi.nlm.nih.gov/geo/) under accession number GSE65998. To validate the transcriptome data, we examined ten representative genes for each of the three mutants by qRT-PCR. The resulting data were correlated with data from the Illumina RNA-Sequencing analysis, and there was no statistically significant difference between the two datasets $(P=0.982)$ (Table 1$)$.

\section{The major low-temperature survival-associated metabolic pathways that were altered in the $\triangle V p a c s p A, \triangle V p a c s p D$ and $\triangle$ VpacspAD mutants}

\section{Major altered metabolic pathways in the $\triangle$ VpacspA mutant} Based on the gene set enrichment analysis (GSEA) of the transcriptome data against the KEGG database, the following seven significantly altered metabolic pathways were identified in the $\triangle V p a c s p A$ mutant at $10^{\circ} \mathrm{C}$ : valine, leucine and isoleucine degradation; the propanoate, ascorbate and aldarate, sulphur, and glycerophospholipid metabolic pathways; ATP-binding cassette (ABC) transporters; and bacterial secretion systems (Table 2). Of these, the DEGs that were linked to valine, leucine and isoleucine degradation as well as propanoate metabolism were upregulated (2.1029- to 8.5787 -fold), which may have resulted in increases in acetyl-CoA and propanoyl-CoA and subsequent entry into the TCA and pyruvate metabolic cycles, respectively, by the $\triangle V p a c s p A$ mutant.

For the other five altered metabolic pathways, most of the DEGs were down-regulated in the $\triangle V p a c s p A$ mutant, which was directly related to its remarkable low-temperature growth inhibition. For example, the expression of twentyfour genes that were linked to $\mathrm{ABC}$ transporters was reduced (0.4917- to 0.1424 -fold); they included the glycine betaine (GB)/proline, oligopeptide, iron(III) and zinc ABC transporters. This indicated the positive regulation of these $\mathrm{ABC}$ transporters by VpaCspA during low-temperature $V$. parahaemolyticus $\mathrm{CHN} 25$ survival.

Bacterial secretion systems play important roles in virulence, symbiosis, interbacterial interactions, and environmental stress [20]. The genes that encoded components of the four secretion system types (T1SS, T2SS, 
Table 1 The qRT-PCR-validated DEGs in the transcriptome data

\begin{tabular}{|c|c|c|c|}
\hline \multirow{2}{*}{$\begin{array}{l}\text { Locus / gene in } \\
\text { V. parahemolyticus CHN25 }\end{array}$} & \multirow[t]{2}{*}{ Description of encoded protein } & \multicolumn{2}{|c|}{ Fold change } \\
\hline & & RNA-Seq & RT-PCR \\
\hline \multicolumn{4}{|l|}{$\Delta V$ pacspa mutant } \\
\hline VpaChn25A_0188 & Hypothetical protein & 13.41 & 10.21 \\
\hline VpaChn25_1642 & Tricarboxylic transport TctC & 10.04 & 10.35 \\
\hline VpaChn25_1640 & GntR family transcriptional regulator & 4.24 & 2.49 \\
\hline VpaChn25A_0561 & Aldehyde dehydrogenase & 7.05 & 5.24 \\
\hline VpaChn25_0068 & Hyperosmotically inducible periplasmic protein & 3.03 & 2.33 \\
\hline VpaChn25A_1398 & L-threonine 3-dehydrogenase & 0.07 & 0.03 \\
\hline VpaChn25A_1399 & 2-amino-3-ketobutyrate CoA ligase & 0.07 & 0.03 \\
\hline VpaChn25_2988 & Amino acid $A B C$ transporter substrate-binding protein & 0.14 & 0.09 \\
\hline VpaChn25A_0149 & Transcriptional regulator $\mathrm{CpxR}$ & 0.22 & 0.12 \\
\hline VpaChn25A_0568 & Transcriptional regulator Betl & 0.48 & 0.32 \\
\hline \multicolumn{4}{|l|}{$\Delta V$ pacspD mutant } \\
\hline VpaChn25_1716 & Glycine betaine transporter periplasmic subunit & 16.04 & 19.13 \\
\hline VpaChn25A_0188 & Hypothetical protein & 12.6 & 11.11 \\
\hline VpaChn25_1642 & Tricarboxylic transport TctC & 7.95 & 7.13 \\
\hline VpaChn25A_0561 & Aldehyde dehydrogenase & 4.08 & 3.23 \\
\hline VpaChn25_1640 & GntR family transcriptional regulator & 2.29 & 2.62 \\
\hline VpaChn25_2248 & Glycerol uptake facilitator protein GlpF & 0.04 & 0.02 \\
\hline VpaChn25A_1398 & L-threonine 3-dehydrogenase & 0.05 & 0.03 \\
\hline VpaChn25A_1399 & 2-amino-3-ketobutyrate CoA ligase & 0.048 & 0.021 \\
\hline VpaChn25_2988 & Amino acid $A B C$ transporter substrate-binding protein & 0.2 & 0.14 \\
\hline VpaChn25A_0149 & Transcriptional regulator $\mathrm{CpxR}$ & 0.3 & 0.25 \\
\hline \multicolumn{4}{|l|}{$\triangle V$ pacspAD mutant } \\
\hline VpaChn25A_1312 & PTS system fructose-specific transporter subunit IIABC & 8.77 & 21.54 \\
\hline VpaChn25A_0303 & PTS system fructose-specific transporter subunit IIA & 8.07 & 19.04 \\
\hline VpaChn25A_1313 & Mannose-6-phosphate isomerase & 5.17 & 16.82 \\
\hline VpaChn25_0669 & Trehalose-6-phosphate hydrolase & 4.51 & 9.77 \\
\hline VpaChn25A_0561 & Aldehyde dehydrogenase & 2.97 & 4.1 \\
\hline VpaChn25_2248 & Glycerol uptake facilitator protein GlpF & 0.033 & 0.039 \\
\hline VpaChn25_2249 & Glycerol kinase & 0.04 & 0.07 \\
\hline VpaChn25_0112 & Phosphoenolpyruvate carboxykinase & 0.17 & 0.22 \\
\hline VpaChn25_2988 & Amino acid $A B C$ transporter substrate-binding protein & 0.31 & 0.43 \\
\hline VpaChn25A_0149 & Transcriptional regulator $\mathrm{CpxR}$ & 0.42 & 0.46 \\
\hline
\end{tabular}

T3SS1 and T6SS2) were identified in the $V$. parahaemolyticus CHN25 genome. Of these, eleven genes were differentially expressed in the $\triangle V p a c s p A$ mutant at the low temperature. Activation of the tolC gene, which encodes an outer membrane protein of T1SS, has been reported in Psychrobacter cryohalolentis K5 during growth at sub-zero temperatures [21]. In this study, tolC gene expression (VpaChn25_1887) was down-regulated (0.4446-fold) in the $\triangle V p a c s p A$ mutant, indicating that VpaCspA positively regulated low-temperature tolC gene expression in $V$. parahaemolyticus CHN25. Likewise, the yscQRSTU genes (VpaChn25A_0952, 0954, 0966, 0967 and 0969), which encode the components of T3SS1, were also highly down-regulated (0.3404- to 0.2109 -fold). However, the expression of five genes that were required for T6SS2 was strongly enhanced (2.4559to 7.0563 -fold) in the $\triangle V p a c s p A$ mutant, which was inconsistent with previous speculation [22]. Future investigations into the biological significance of the secretion systems and their differential expression characteristics 
Table 2 Major altered metabolic pathways in the $\triangle V$ pacspA, $\triangle V$ pacspD and $\triangle V$ pacspAD mutants of $V$. parahaemolyticus CHN25 grown at the low temperature

\begin{tabular}{|c|c|c|c|}
\hline Changed metabolic pathway & Locus / Gene* & Fold change & Description of encoded protein \\
\hline \multicolumn{4}{|l|}{$\Delta$ V pacspA mutant } \\
\hline \multirow{11}{*}{$\begin{array}{l}\text { Valine, leucine and isoleucine } \\
\text { degradation }\end{array}$} & VpaChn25A_0480 & 2.4983 & Acetyl-CoA acetyltransferase \\
\hline & VpaChn25A_0554 & 3.0843 & Hydroxymethylglutaryl-CoA lyase \\
\hline & VpaChn25A_0555 & 3.2361 & Acyl-CoA carboxylase alpha chain \\
\hline & VpaChn25A_0556 & 2.4095 & Enoyl-CoA hydratase/isomerase \\
\hline & VpaChn25A_0557 & 2.662 & Acyl-CoA carboxyltransferase beta chain \\
\hline & VpaChn25A_0558 & 2.216 & Acyl-CoA dehydrogenase \\
\hline & VpaChn25A_0560 & 2.8734 & Acyl-CoA thiolase \\
\hline & VpaChn25A_0561 & 7.0543 & Aldehyde dehydrogenase \\
\hline & VpaChn25A_0565 & 7.3736 & 3-hydroxyisobutyrate dehydrogenase \\
\hline & VpaChn25A_1033 & 2.7479 & Methylmalonate-semialdehyde dehydrogenase \\
\hline & VpaChn25A_1036 & 2.1029 & Acyl-CoA dehydrogenase \\
\hline \multirow[t]{5}{*}{ Propanoate metabolism } & VpaChn25_1376 & 6.0834 & 4-aminobutyrate aminotransferase \\
\hline & VpaChn25_1635 & 6.3526 & PrpE protein \\
\hline & VpaChn25_1638 & 8.5787 & Methylcitrate synthase \\
\hline & VpaChn25_1639 & 6.7065 & 2-methylisocitrate lyase \\
\hline & VpaChn25_2798 & 3.959 & Acetyl-CoA synthetase \\
\hline \multirow[t]{25}{*}{ ABC transporters } & VpaChn25A_0128 & 0.4376 & $A B C$ transporter substrate-binding protein \\
\hline & VpaChn25A_0130 & 0.428 & ABC transporter ATP-binding protein \\
\hline & VpaChn25A_0533 & 3.1521 & $\begin{array}{l}\text { High-affinity branched-chain amino acid transport } \\
\text { ATP-binding protein }\end{array}$ \\
\hline & VpaChn25A_0535 & 3.9903 & $A B C$ transporter membrane spanning protein \\
\hline & VpaChn25A_0536 & 5.0642 & High-affinity branched-chain amino acid transport permease \\
\hline & VpaChn25A_0537 & 6.8795 & Hypothetical protein \\
\hline & VpaChn25A_0538 & 4.7093 & $\begin{array}{l}\text { High-affinity branched-chain amino acid transport } \\
\text { ATP-binding protein }\end{array}$ \\
\hline & VpaChn25A_0571 & 0.3015 & Glycine betaine-binding $A B C$ transporter \\
\hline & VpaChn25A_0572 & 0.3358 & Permease \\
\hline & VpaChn25A_0573 & 0.3801 & ABC transporter ATP-binding protein \\
\hline & VpaChn25A_0595 & 0.4067 & Ribose $A B C$ transporter permease \\
\hline & VpaChn25A_0604 & 0.406 & Iron (III) $A B C$ transporter periplasmic iron-compound-binding protein \\
\hline & VpaChn25A_1325 & 0.2076 & Iron (III) ABC transporter ATP-binding protein \\
\hline & VpaChn25A_1326 & 0.2262 & Iron (III) $A B C$ transporter periplasmic iron-compound-binding protein \\
\hline & VpaChn25A_1327 & 0.3019 & Iron-hydroxamate transporter permease subunit \\
\hline & VpaChn25A_1333 & 2.1634 & Transport protein \\
\hline & VpaChn25A_1544 & 0.2026 & Iron-dicitrate transporter substrate-binding subunit \\
\hline & VpaChn25_0306 & 0.4761 & Thiamine transporter membrane protein \\
\hline & VpaChn25_0363 & 2.1743 & $A B C$ transporter substrate binding protein \\
\hline & VpaChn25_0846 & 0.4129 & Zinc $A B C$ transporter permease \\
\hline & VpaChn25_0848 & 0.4556 & Zinc $A B C$ transporter periplasmic zinc-binding protein \\
\hline & VpaChn25_1344 & 0.2076 & Oligopeptide ABC transporter ATP-binding protein \\
\hline & VpaChn25_1345 & 0.2716 & Oligopeptide ABC transporter ATP-binding protein \\
\hline & VpaChn25_1346 & 0.2355 & Oligopeptide ABC transporter permease \\
\hline & VpaChn25_1347 & 0.3245 & Oligopeptide $A B C$ transporter permease \\
\hline
\end{tabular}


Table 2 Major altered metabolic pathways in the $\triangle V$ pacspA, $\triangle V$ pacspD and $\triangle V$ pacspAD mutants of $V$. parahaemolyticus CHN25 grown at the low temperature (Continued)

\begin{tabular}{|c|c|c|c|}
\hline & VpaChn25_1348 & 0.3723 & Oligopeptide $A B C$ transporter periplasmic oligopeptide-binding protein \\
\hline & VpaChn25_1613 & 2.4584 & Amino acid $A B C$ transporter substrate-binding protein \\
\hline & VpaChn25_1714 & 0.4917 & Glycine/betaine/proline $A B C$ transporter \\
\hline & VpaChn25_1913 & 2.222 & Hypothetical protein \\
\hline & VpaChn25_2428 & 0.4529 & Iron (III) $\mathrm{ABC}$ transporter permease \\
\hline & VpaChn25_2429 & 0.3234 & Iron (III) ABC transporter periplasmic iron-compound-binding protein \\
\hline & VpaChn25_2987 & 0.2357 & Amino acid $A B C$ transporter permease \\
\hline & VpaChn25_2988 & 0.1424 & Amino acid $A B C$ transporter substrate-binding protein \\
\hline Bacterial secretion systems & VpaChn25A_0952 & 4.5459 & Hypothetical protein \\
\hline & VpaChn25A_0954 & 6.1398 & ClpA / B-type chaperone \\
\hline & VpaChn25A_0966 & 6.0405 & Hypothetical protein \\
\hline & VpaChn25A_0967 & 7.0563 & Hypothetical protein \\
\hline & VpaChn25A_0969 & 2.4559 & Hypothetical protein \\
\hline & VpaChn25_1662 & 0.3404 & Type III secretion system protein \\
\hline & VpaChn25_1663 & 0.2481 & Type III secretion system protein \\
\hline & VpaChn25_1664 & 0.2099 & Translocation protein in type III secretion \\
\hline & VpaChn25_1665 & 0.2746 & Translocation protein in type III secretion \\
\hline & VpaChn25_1666 & 0.2109 & Translocation protein in type III secretion \\
\hline & VpaChn25_1887 & 0.4446 & Outer membrane protein TolC \\
\hline Ascorbate and aldarate metabolism & VpaChn25A_0230 & 0.1973 & PTS system ascorbate-specific transporter subunit IIC \\
\hline & VpaChn25A_0231 & 0.1668 & Sugar phosphotransferase component II B \\
\hline & VpaChn25A_0232 & 0.1096 & Phosphotransferase enzyme II, A component \\
\hline Sulfur metabolism & VpaChn25_0788 & 0.2523 & Cysteine synthase A \\
\hline & VpaChn25_0937 & 3.0238 & Cysteine synthase / cystathionine beta-synthase family protein \\
\hline & VpaChn25_1397 & 2.7136 & Homoserine O-succinyltransferase \\
\hline & VpaChn25_2650 & 0.4424 & Phosphoadenosine phosphosulfate reductase \\
\hline & VpaChn25_2651 & 0.4934 & Sulfite reductase subunit beta \\
\hline & VpaChn25_2652 & 0.2017 & Sulfite reductase (NADPH) flavoprotein subunit alpha \\
\hline & VpaChn25_2692 & 2.1317 & Cystathionine gamma-synthase \\
\hline Glycerophospholipid metabolism & VpaChn25A_0425 & 0.4957 & Diacylglycerol kinase \\
\hline & VpaChn25A_0732 & 2.2688 & Outer membrane phospholipase A \\
\hline & VpaChn25_0642 & 0.4375 & Phosphatidylglycerophosphatase A \\
\hline & VpaChn25_0885 & 0.3795 & Surfactin synthetase \\
\hline & VpaChn25_2245 & 0.2175 & Glycerophosphodiester phosphodiesterase \\
\hline & VpaChn25_2251 & 0.2013 & Glycerol-3-phosphate dehydrogenase \\
\hline$\Delta V$ pacspD mutant & & & \\
\hline PTS & VpaChn25A_0230 & 0.2003 & PTS system ascorbate-specific transporter subunit IIC \\
\hline & VpaChn25A_0231 & 0.1886 & Sugar phosphotransferase component II B \\
\hline & VpaChn25A_0232 & 0.1253 & Phosphotransferase enzyme II, A component \\
\hline & VpaChn25A_0303 & 12.8135 & PTS system fructose-specific transporter subunit IIA \\
\hline & VpaChn25A_1196 & 0.3193 & Mannitol-specific PTS system enzyme II component \\
\hline & VpaChn25A_1309 & 3.4141 & PTS system fructose-specific transporter subunit IIB \\
\hline & VpaChn25A_1312 & 4.9223 & PTS system fructose-specific transporter subunit IIABC \\
\hline & VpaChn25_0668 & 0.4181 & PTS system trehalose (maltose)-specific transporter subunits IIBC \\
\hline
\end{tabular}


Table 2 Major altered metabolic pathways in the $\Delta V$ pacspA, $\triangle V p a c s p D$ and $\triangle V$ pacspAD mutants of $V$. parahaemolyticus CHN25 grown at the low temperature (Continued)

\begin{tabular}{|c|c|c|c|}
\hline & VpaChn25_2564 & 2.1655 & PTS system cellobiose-specific transporter subunit IIA \\
\hline & VpaChn25_2566 & 2.8755 & PTS system cellobiose-specific transporter subunit IIB \\
\hline \multirow{11}{*}{$\begin{array}{l}\text { Alanine, aspartate and glutamate } \\
\text { metabolism }\end{array}$} & VpaChn25A_0370 & 3.4733 & Adenylosuccinate synthase \\
\hline & VpaChn25_0104 & 2.2225 & Glutamine synthetase \\
\hline & VpaChn25_0345 & 0.3501 & Glucosamine-fructose-6-phosphate aminotransferase \\
\hline & VpaChn25_0436 & 2.1782 & Glutamate synthase subunit beta \\
\hline & VpaChn25_0437 & 2.0222 & Glutamate synthase subunit alpha \\
\hline & VpaChn25_1375 & 3.4554 & Succinate-semialdehyde dehydrogenase \\
\hline & VpaChn25_1376 & 3.0672 & 4-aminobutyrate aminotransferase \\
\hline & VpaChn25_2552 & 0.4804 & Glutaminase \\
\hline & VpaChn25_2583 & 2.0986 & Aspartate carbamoyltransferase \\
\hline & VpaChn25_2584 & 2.7419 & Aspartate carbamoyltransferase \\
\hline & VpaChn25_2784 & 0.4744 & Aspartate ammonia-lyase \\
\hline \multirow[t]{8}{*}{ Arginine and proline metabolism } & VpaChn25_1371 & 2.2205 & Aldehyde dehydrogenase \\
\hline & VpaChn25_1372 & 2.779 & Oxidoreductase \\
\hline & VpaChn25_1373 & 3.3592 & Carbon-nitrogen hydrolase \\
\hline & VpaChn25_1374 & 3.206 & Hypothetical protein \\
\hline & VpaChn25_2581 & 3.3917 & Arginine deiminase \\
\hline & VpaChn25_2719 & 2.8035 & Succinylglutamic semialdehyde dehydrogenase \\
\hline & VpaChn25_2720 & 3.9099 & Arginine/ornithine succinyltransferase \\
\hline & VpaChn25_2721 & 2.1486 & $\begin{array}{l}\text { Bifunctional N-succinyldiaminopimelate-aminotransferase/ } \\
\text { acetylornithine transaminase protein }\end{array}$ \\
\hline \multirow{8}{*}{$\begin{array}{l}\text { Valine, leucine and isoleucine } \\
\text { degradation }\end{array}$} & VpaChn25A_0480 & 3.4643 & Acetyl-CoA acetyltransferase \\
\hline & VpaChn25A_0555 & 2.023 & Acyl-CoA carboxylase alpha chain \\
\hline & VpaChn25A_0557 & 2.0591 & Acyl-CoA carboxyltransferase beta chain \\
\hline & VpaChn25A_0561 & 4.081 & Aldehyde dehydrogenase \\
\hline & VpaChn25A_0565 & 3.2953 & 3-hydroxyisobutyrate dehydrogenase \\
\hline & VpaChn25A_1038 & 0.4859 & Enoyl-CoA hydratase / isomerase \\
\hline & VpaChn25_0020 & 2.6571 & Multifunctional fatty acid oxidation complex subunit alpha \\
\hline & VpaChn25_2076 & 0.4816 & 3-ketoacyl-CoA thiolase \\
\hline \multirow[t]{5}{*}{ Propanoate metabolism } & VpaChn25_1635 & 2.316 & PrpE protein \\
\hline & VpaChn25_1638 & 4.6552 & Methylcitrate synthase \\
\hline & VpaChn25_1639 & 3.654 & 2-methylisocitrate lyase \\
\hline & VpaChn25_2433 & 3.0153 & Bifunctional aconitate hydratase 2/2-methylisocitrate dehydratase \\
\hline & VpaChn25_2798 & 2.1035 & Acetyl-CoA synthetase \\
\hline \multirow[t]{5}{*}{ Nitrogen metabolism } & VpaChn25A_0296 & 4.3124 & Oxidoreductase protein \\
\hline & VpaChn25A_0917 & 3.7442 & Nitrite reductase large subunit \\
\hline & VpaChn25A_1392 & 0.4447 & Carbonic anhydrase \\
\hline & VpaChn25_1817 & 9.4056 & Cytochrome c552 \\
\hline & VpaChn25_2448 & 0.2912 & Carbonic anhydrase \\
\hline \multicolumn{4}{|l|}{$\triangle V$ pacspAD mutant } \\
\hline \multirow[t]{3}{*}{ TCA } & VpaChn25_0313 & 0.2603 & Malate dehydrogenase \\
\hline & VpaChn25_0837 & 0.1445 & Type II citrate synthase \\
\hline & VpaChn25_0838 & 0.2716 & Succinate dehydrogenase cytochrome b556 large membrane subu \\
\hline
\end{tabular}


Table 2 Major altered metabolic pathways in the $\triangle V$ pacspA, $\triangle V$ pacspD and $\triangle V$ pacspAD mutants of $V$. parahaemolyticus CHN25 grown at the low temperature (Continued)

\begin{tabular}{|c|c|c|c|}
\hline & VpaChn25_0840 & 0.402 & Succinate dehydrogenase flavoprotein subunit \\
\hline & VpaChn25_0841 & 0.3554 & Succinate dehydrogenase iron-sulfur subunit \\
\hline & VpaChn25_0843 & 0.3915 & Dihydrolipoamide succinyltransferase \\
\hline & VpaChn25_0844 & 0.3535 & Succinyl-CoA synthetase subunit beta \\
\hline & VpaChn25_0845 & 0.3009 & Succinyl-CoA synthetase subunit alpha \\
\hline & VpaChn25_1035 & 0.4921 & Isocitrate dehydrogenase \\
\hline & VpaChn25_2762 & 0.478 & Fumarate reductase flavoprotein subunit \\
\hline & VpaChn25_2763 & 0.4836 & Fumarate reductase iron-sulfur subunit \\
\hline & VpaChn25_2764 & 0.4898 & Fumarate reductase subunit $C$ \\
\hline & VpaChn25_2765 & 0.3058 & Fumarate reductase subunit $D$ \\
\hline \multirow[t]{8}{*}{ PTS } & VpaChn25A_0230 & 0.3036 & PTS system ascorbate-specific transporter subunit IIC \\
\hline & VpaChn25A_0231 & 0.2665 & Sugar phosphotransferase component II B \\
\hline & VpaChn25A_0232 & 0.232 & Phosphotransferase enzyme II, A component \\
\hline & VpaChn25A_0303 & 8.0738 & PTS system fructose-specific transporter subunit IIA \\
\hline & VpaChn25A_1312 & 8.7743 & PTS system fructose-specific transporter subunit IIABC \\
\hline & VpaChn25_0356 & 0.3818 & PTS system mannitol-specific transporter subunit IIABC \\
\hline & VpaChn25_2566 & 0.3343 & PTS system cellobiose-specific transporter subunit IIB \\
\hline & VpaChn25_0668 & 3.948 & PTS system trehalose (maltose)-specific transporter subunits IIBC \\
\hline \multirow[t]{2}{*}{ Butanoate metabolism } & VpaChn25A_0528 & 2.6111 & Acetoacetyl-CoA synthetase \\
\hline & VpaChn25A_0560 & 2.8181 & Acyl-CoA thiolase \\
\hline \multirow[t]{2}{*}{ Fructose and mannose metabolism } & VpaChn25A_1313 & 5.1663 & Mannose-6-phosphate isomerase \\
\hline & VpaChn25_0355 & 0.3626 & Mannitol-1-phosphate 5-dehydrogenase \\
\hline \multirow[t]{6}{*}{ Pyruvate metabolism } & VpaChn25A_0307 & 2.3933 & Hypothetical protein \\
\hline & VpaChn25A_0367 & 0.321 & Phosphoenolpyruvate synthase \\
\hline & VpaChn25A_0934 & 0.4136 & D-lactate dehydrogenase \\
\hline & VpaChn25_0112 & 0.1659 & Phosphoenolpyruvate carboxykinase \\
\hline & VpaChn25_1693 & 2.2321 & Aldehyde dehydrogenase \\
\hline & VpaChn25_1927 & 0.408 & Pyruvate kinase II \\
\hline \multirow[t]{3}{*}{ Oxidative phosphorylation } & VpaChn25A_0546 & 2.1237 & Cytochrome BD2 subunit II \\
\hline & VpaChn25_1519 & 0.4848 & Cytochrome c oxidase subunit CcoP \\
\hline & VpaChn25_1521 & 0.4651 & cbb3-type cytochrome c oxidase subunit II \\
\hline \multirow{6}{*}{$\begin{array}{l}\text { Cysteine and methionine } \\
\text { metabolism }\end{array}$} & VpaChn25_0576 & 0.293 & Homocysteine synthase \\
\hline & VpaChn25_0788 & 0.2948 & Cysteine synthase A \\
\hline & VpaChn25_0937 & 2.3269 & Cysteine synthase/cystathionine beta-synthase family protein \\
\hline & VpaChn25_1880 & 2.8826 & $\begin{array}{l}\text { 5-methyltetrahydropteroyltriglutamate-homocysteine } \\
\text { S-methyltransferase }\end{array}$ \\
\hline & VpaChn25_2471 & 0.4433 & S-ribosylhomocysteinase \\
\hline & VpaChn25_2646 & 2.2961 & Aspartate kinase \\
\hline \multirow[t]{6}{*}{ Arginine and proline metabolism } & VpaChn25A_1611 & 0.4145 & $\begin{array}{l}\text { Bifunctional proline dehydrogenase/pyrroline-5-carboxylate } \\
\text { dehydrogenase }\end{array}$ \\
\hline & VpaChn25_1332 & 0.1954 & Hydroxyproline-2-epimerase \\
\hline & VpaChn25_1335 & 0.3527 & Ornithine cyclodeaminase \\
\hline & VpaChn25_1544 & 0.3291 & NAD-glutamate dehydrogenase \\
\hline & VpaChn25_2719 & 0.3969 & Succinylglutamic semialdehyde dehydrogenase \\
\hline & VpaChn25_2720 & 0.482 & Arginine /ornithine succinyltransferase \\
\hline
\end{tabular}


Table 2 Major altered metabolic pathways in the $\triangle V$ pacspA, $\triangle V$ pacspD and $\triangle V$ pacspAD mutants of $V$. parahaemolyticus CHN25 grown at the low temperature (Continued)

\begin{tabular}{llll}
\hline & VpaChn25_2721 & 0.2687 & $\begin{array}{l}\text { Bifunctional N-succinyldiaminopimelate-aminotransferase / } \\
\text { acetylornithine transaminase protein }\end{array}$ \\
$\begin{array}{llll}\text { Alanine, aspartate and glutamate } \\
\text { metabolism }\end{array}$ & $\begin{array}{l}\text { VpaChn25_0345 } \\
\text { Glucosamine-fructose-6-phosphate aminotransferase }\end{array}$ & 0.2743 & Glutamate synthase subunit beta \\
& VpaChn25_0438 & 2.461 & Glutamate synthase, large subunit \\
& VpaChn25_0439 & 2.6963 & Alanine dehydrogenase \\
& VpaChn25_2022 & 0.4771 & Cytoplasmic asparaginase I \\
\hline
\end{tabular}

"VpaChn25, chromosome 1; VpaChn25A, chromosome 2

during low-temperature survival by $V$. parahaemolyticus will provide important insights on this topic.

\section{Major altered metabolic pathways in the $\Delta V p a c s p D$ mutant}

Based on the GESA-KEGG analysis, the following six significantly altered metabolic pathways were identified in the $\triangle$ VpacspD mutant at $10{ }^{\circ} \mathrm{C}$ : the phosphotransferase system (PTS); alanine, aspartate and glutamate metabolism; arginine and proline metabolism; the propanoate and nitrogen metabolic pathways; valine, leucine and isoleucine degradation.

Consistent with its active low-temperature growth phenotype, several DEGs that were linked to PTS, to nitrogen, arginine and proline metabolism and to alanine, aspartate and glutamate metabolism were significantly up-regulated in the $\Delta V p a c s p D$ mutant. A major barrier to protein function at low temperatures is the inability to maintain sufficient flexibility so that it can increase its interactions with substrates to reduce its required activation energy [23]. In arginine and proline metabolism, all eight DEGs were up-regulated in the $\triangle V$ pacspD mutant. For example, expression of an arginine deiminase (VpaChn25_2581) and an arginine/ornithine succinyltransferase (VpaChn25_2720), which are required to convert L-arginine to L-citrulline and then to N2succinyl-L-arginine, were up-regulated by 3.3917- and 3.9099-fold, respectively. Arginines are structurally stabilizing factors that contain side chains that form salt bridges and hydrogen bonds [24]. Our data indicated that a low-temperature decrease in $\mathrm{L}$-arginine in the $\triangle$ VpacspD mutant may have promoted increased protein flexibility. Moreover, the abundance of proline residues is related to increased protein stability due to the rigidity of the N-C $\alpha$ bond [23]. In this study, a decrease in proline resulted from up-regulated proline metabolismassociated enzymes may have also enhanced protein flexibility in the $\triangle$ VpacspD mutant. To our knowledge, these genes have not been previously linked to lowtemperature survival.

Expression of a glutamine synthetase (VpaChn25_0104), which catalyses L-glutamate to L-glutamine, was up-regulated in the alanine, aspartate and glutamate metabolic pathways. However, the genes that encoded a glutaminase (VpaChn25_2552) and a glucosamine-fructose6-phosphate aminotransferase (VpaChn25_0345), which convert L-glutamine to L-glutamate and then to $\mathrm{D}$ glucosamine, showed opposite expression profiles, which suggested a decrease in L-glutamate accumulation in the $\Delta$ VpacspD mutant. This was also suppressed in the psycrophilic proteins of Vibrio salmonicida [25].

Unexpectedly, the comparative transcriptome analysis revealed very few genes that were up-regulated in $\Delta$ VpacspD but down-regulated in the $\Delta$ VpacspA mutant, indicating that these genes were specifically and negatively governed by $V p a C s p D$. Additionally, in the $\triangle V p a c s p A$ mutant, VpacspD gene expression was increased (2.5073-fold) at the low temperature, which was validated by qRT-PCR analysis, but no significant change in VpacspA gene expression was observed in the $\triangle V$ pacspD mutant. The results indicated that $V p a C s p D$ was inhibited by $V p a C s p A$ at low temperatures, which was consistent with the growth phenotypes described above.

\section{Major altered metabolic pathways in the $\triangle V p a c s p A D$ mutant}

Similarly, the GESA-KEGG analysis revealed the following nine significantly changed metabolic pathways in the $\triangle$ Vpacsp $A D$ mutant at $10^{\circ} \mathrm{C}$ : TCA; PTS; butanoate metabolism; fructose and mannose metabolism; the pyruvate and the cysteine and methionine metabolic pathways; arginine and proline metabolism; alanine, aspartate and glutamate metabolism; oxidative phosphorylation. Interestingly, these altered metabolic pathways were different from those that were induced in the $\triangle V p a c s p A$ mutant, although both mutants demonstrated the slower-growth phenotype at the low temperature. Most of the DEGs that were linked to TCA, oxidative phosphorylation, and pyruvate metabolism were inhibited in the $\triangle V p a c s p A D$ mutant, which may explain its slower growth at this low temperature. The down-regulated central metabolic pathways were also observed in other bacteria that were grown at a low temperature [26]. 
Similar to the $\Delta V p a c s p D$ mutant, the alanine, aspartate and glutamate metabolic pathways, PTS, and the arginine and proline metabolic pathways were also significantly changed in the $\triangle V$ pacsp $A D$ mutant. However, distinct expression patterns were detected in the two mutants. For example, in contrast to the $\Delta V \operatorname{pacspD}$ mutant, all seven DEGs that were involved in arginine and proline metabolism were down-regulated (0.482- to 0.1954-fold) in the $\triangle V$ pacsp $A D$ mutant. Additionally, the phosphoenolpy ruvate-dependent PTS is a major sugar transport multicomponent system in bacteria, by which multiple sugars are transported into bacteria, concomitantly phosphorylated, and fed into glycolysis [27]. In this study, expression of the genes that encoded the cellobiose- and trehalose (maltose)-specific transporter subunits (VpaChn25_2566 and Chn25_0668) also displayed opposite patterns between the $\triangle V p a c s p A D$ and $\triangle V p a c s p D$ mutants. These results highlighted the antagonistic regulatory effects by $V p a C s p A$ and $V p a C s p D$ on low-temperature survival of $V$. parahaemolyticus CHN25.

In cysteine and methionine metabolism, a homocysteine synthase (VpaChn25_0576) and S-ribosylhomocysteinase (VpaChn25_2471), which are involved in converting O-ace tyl-L-homoserine and S-ribosyl-L-homocysteine to L-homo cysteine, were down-regulated (0.2930- and 0.4433-fold, respectively) in the $\triangle V$ pacsp $A D$ mutant. However, a 5-methyl tetrahydropteroyltriglutamate-homocysteine S-methyltransf erase (VpaChn25_1880) that catalyses L-homocysteine to L-methionine was up-regulated (2.8826-fold). These results suggest the attenuation of L-homocysteine in the $\triangle V$ pacspAD mutant, which may reduce interference by L-homocysteine with amino acid metabolic and translation processes at low temperatures [28].

\section{Differentially expressed regulators (DERs) that are involved in the low-temperature survival of the $\triangle V p a c s p A$, $\triangle V p a c s p D$ and $\triangle V p a c s p A D$ mutants}

The $V$. parahaemolyticus $\mathrm{CHN} 25$ genome contains approximately two hundred and seventy-two genes that encode putative transcriptional or response regulators, which represent approximately $5.8 \%$ of all proteinencoding genes in the bacterium. Changes in the expression of transcription factors, especially the master regulators, can modulate global regulatory networks that, in some cases, are essential for bacterial adaptation to changing environments [13]. In this study, the genome-level transcriptome data revealed thirty, twentythree and thirty-six DERs in the $\triangle V \operatorname{Vacsp} A, \triangle V p a c s p D$ and $\triangle$ Vpacsp $A D$ mutants at $10{ }^{\circ} \mathrm{C}$, respectively (see Additional file 3: Table S1). They globally or specifically regulate various cellular processes, including cold-tempe rature survival in bacteria, by regulating transcriptional or response regulators that are involved in DNA-binding,
LysR-type transcriptional regulators, and GntR, AraC/XylS, ArsR, LuxR, and DeoR regulator families.

Of these regulators, several directly regulate gene expression in response to environmental signals in other bacteria. For example, a recombination regulator, RecX (VpaChn25_2483), which regulates DNA recombination and protects the cell from ionising radiation and UVirradiation in E. coli [29], was notably down-regulated (0.2654-fold) in the $\Delta V p a c s p A$ mutant; this indicates the positive regulation of RecX by $V p a C s p A$ in $V$. parahaemolyticus $\mathrm{CHN} 25$ at low temperatures. Interestingly, a transcriptional regulator, BetI (VpaChn25A_0568), was also inhibited in the $\triangle V$ pacsp $A$ mutant (0.4784-fold), which negatively regulated the $\operatorname{bet} T$ and $\operatorname{bet} I B A$ genes that governed GB synthesis from choline in E. coli [30]. Moreover, two genes (VpaChn25_1793 and Chn25_1442), which encode the osmotically inducible betaine-cholinecarnitine transporters (BCCTs) that mediate the acquisition of preformed $\mathrm{GB}$ [31], were also down-regulated in the $\triangle V p a c s p A$ mutant. These data indicate that $V p a C s p A$ may stimulate an accumulation of cellular GB that adjusts the hydration level of the bacterial cell cytoplasm at low temperatures [32, 33]. Additionally, expression of an important transcriptional regulator, PdhR (VpaChn25_2454), which belongs to the GntR family of transcriptional regulators, was repressed $(0.3122$-fold $)$ in the $\Delta$ Vpacsp $A$ mutant. PdhR regulates central metabolism by controlling transcription of the components that form the pyruvate dehydrogenase complex [34].

Among the DERs that were elicited in the $\triangle$ VpacspD mutant, two regulators (a response regulator (VpaChn25_1251) and a MerR family transcriptional regulator (VpaChn25A_1361)) were up-regulated in the $\triangle V$ pacspD mutant at low temperatures (2.891- and 2.8939-fold, respectively). The latter regulates gene transcription in response to different environmental signals, including signals from heavy metal ions, organic compounds, and oxidative stress [35]. Approximately 56.5\% of the DERs in the $\triangle V$ pacspD mutant were down-regulated, of which half were exclusively expressed in the $\Delta V p a c s p D$ mutant (e.g., the two-component response regulator (VpaChn25A_1000) and sigma-E factor negative regulatory protein RseA (VpaChn25_2510)) [36].

Transcriptome data comparisons revealed mosaic DER expression profiles in the VpacspAD mutant. Interestingly, three regulators of T3SS1 gene expression were inhibited in both $\triangle V p a c s p D$ and $\triangle V p a c s p A D$ cells at the low temperature. These included ExsA (VpaChn25_1689) and ExsE (VpaChn25_1692), which belonged to the ExsACDE regulatory cascade, and a T3SS1 regulator (VpaChn25_1651), which was indicative of positive regulation of T3SS1 by $V p a C s p D$ at low temperatures; this function was similar to that of $V p a C s p A$. Likewise, expression of the UhpC regulator (VpaChn25A_0772), 
a membrane-bound sensor for external glucose-6phosphate in E. coli [37], was also decreased in the two mutants. UhpC was reported to negatively modulate a YE0480 gene in Yersinia enterocolitica, which encoded a homologue of the FhaC accessory protein; $\mathrm{FhaC}$ was strongly expressed at $10{ }^{\circ} \mathrm{C}$ but not at $37{ }^{\circ} \mathrm{C}$ in Bordetella pertussis [38].

Interestingly, three DERs were detected in all three mutants, and the other five were synchronously induced in both $\triangle V p a c s p A$ and $\triangle V p a c s p D$ cells, indicating either similar regulatory functions that were shared between $V p a C \operatorname{spA}$ and $V p a C s p D$ or $V p a C s p A / D$-independent regulation in $V$. parahaemolyticus $\mathrm{CHN} 25$ at low temperatures. The molecular responses of bacteria to external environmental signals are complex, but twocomponent signal transduction systems reportedly play important roles in low-temperature adaptation by several bacteria [39-41]. In this study, the expression of a cytosolic response regulator, CpxR (VpaChn25A_0149), which belongs to the two-component $\mathrm{Cpx}$-envelope stress system [42], was repressed in the three mutants. The Cpx system responds to a broad range of environmental stimuli (e.g., pH, salt, metals, lipids and misfolded proteins) that cause perturbation of the envelope [43]. In this study, our data showed positive regulation of CpxR by both $V p a C s p A$ and $V p a C s p D$, which may have protected envelope-bound proteins from lowtemperature damage.

Taken together, our transcriptome data revealed a complex molecular regulatory network that was specifically, coordinately or antagonistically modulated by $V p a C s p A$ and $V p a C s p D$ during low-temperature adaptation by $V$. parahaemolyticus. Numerous regulators, which act as activators or repressors in response to multiple environmental stressors in bacteria, were also elicited in the three mutants. A future in-depth regulatory network analysis will improve our understanding of low-temperature adaptation mechanisms in $V$. parahaemolyticus.

\section{Possible low-temperature adaptation mechanisms that are mediated by VpaCspA and VpaCspD in V. parahaemolyticus CHN25}

The most common strategy that has been adopted by bacteria to survive a low-temperature environment is the accumulation of compatible solutes (e.g., GB, choline, carnitine, and mannitol) by uptake or biosynthesis [11]. In this study, a similar low-temperature strategy by $V$. parahaemolyticus CHN25 was observed (Fig. 4). For example, seven genes that were associated with GB biosynthesis, $\mathrm{BCCT}$ and GB-binding $\mathrm{ABC}$ transporters were significantly inhibited in the $\triangle V p a c s p A$ mutant, which indicated that $V p a C s p A$ likely stimulated cellular GB accumulation to adjust the hydration level of the cytoplasm and to protect the bacterium from low-temperature damage.

Interestingly, in this study, the glycerophospholipid metabolism-associated $g l p D F K Q$ genes were more strongly inhibited in $\triangle V p a c s p A D$ than in the $\triangle V p a c s p A$ or $\triangle V p a c s p D$ mutants, which indicated a coordinated low-temperature activation of the genes by $V p a C s p A$ and $V p a C s p D$. For example, expression of the $g l p F$ gene (VpaChn25_2248), which encodes a glycerol uptake facilitator and functions in substrate equilibration between the extracellular and intracellular spaces [44], was down-regulated in $\triangle$ VpacspA (0.2794-fold), strongly

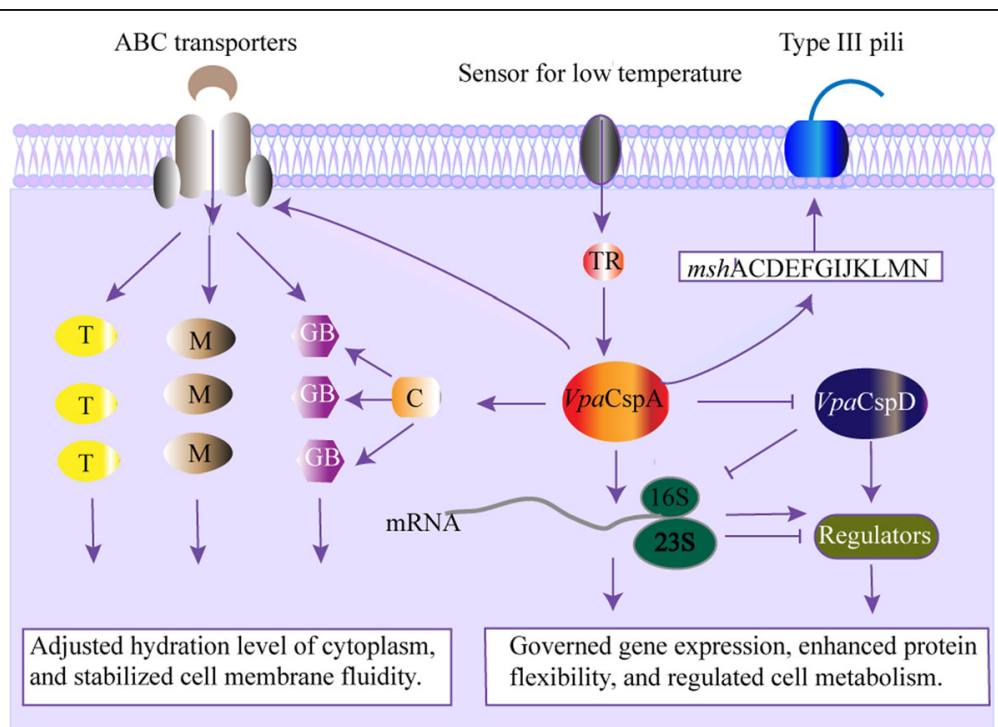

Fig. 4 The possible VpaCspA and VpaCspD-mediated molecular mechanisms that underlie low-temperature adaptation by $V$. parahaemolyticus CHN25. T, trehalose; M, mannitol; GB, glycine betaine; C, CAMP regulator protein; TR, transducer; $16 \mathrm{~S}$ and 23S, rRNA subunits 
suppressed in $\triangle V p a c s p D$ (0.0457-fold), and suppressed in $\triangle V p a c s p A D$ (0.0337-fold). Similarly, the $g l p Q$ and glpD genes encode a glycerophosphodiester phosphodiesterase (VpaChn25_2245) and a glycerol-3-phosphate dehydrogenase (VpaChn25_2251), and they catalyse sn-glycero-3-phosphocholine to choline and sn-glycerol3-phosphate (G3P) and G3P to dihydroxyacetone phosphate (DHAP), respectively. Expression of the $g l p Q$ and $g l p D$ genes was also more strongly inhibited in $\triangle V p a c$ spAD (0.0787- and 0.0636-fold, respectively) than in $\triangle$ VpacspA (0.2175- and 0.2013-fold, respectively) or $\triangle$ VpacspD (0.1011- and 0.0302-fold, respectively), indicating a positively superposed regulation of the choline biosynthesis genes by $V p a C s p A$ and $V p a C s p D$; this may have resulted in an increase in cellular compatible solutes to maintain cell membrane integrity at low temperatures. However, the decreased DHAP indirectly led to increased biofilm formation and contributed to several survival advantages under various environmental and energy insults in several other bacteria $[45,46]$. Moreover, the $g l p K$ gene, which encodes a glycerol kinase (VpaChn25_2249) that catalyses glycerol to G3P, showed similar expression profiles in all three mutants, which probably resulted in attenuated cellular G3P accumulation at low temperatures. G3P has been reported to mediate catabolite repression through adenylate cyclase inhibition, which leads to decreases in $3^{\prime}-5^{\prime}$-cyclic adenosine monophosphate (cAMP) and inactivation of the cAMP receptor protein (CRP); CRP is a global regulator that participates in sugar metabolism and plays an important role in cold adaptation by E. coli [44].

Protective roles for trehalose in response to lowtemperature, heat and osmotic stressors have been reported, including prevention of the denaturation and aggregation of specific proteins, in vivo activity as a free radical scavenger, and stabilisation of cell membrane fluidity [47]. In this study, expression of the trehalose (maltose)-specific transporter subunit II BC components (VpaChn25_0668) was down-regulated in the $\triangle V p a c s p A$ (0.2935-fold) and $\triangle$ VpacspD (0.4181-fold) mutants, indicating the positive regulation of trehalose-specific transport by $V p a C s p A$ and $V p a C \operatorname{sp} D$ to promote bacterial adaptation to a low-temperature environment. Nevertheless, the gene showed an opposite expression pattern in the $\triangle V p a c s p A D$ mutant (3.948-fold), which implied unknown regulatory mechanisms in the $\triangle$ VpacspAD mutant by which trehalose was transported.

Biofilm formation is related to bacterial survival in various environments. It has been reported that type IV pili (TFP) played an important role in the biofilm formation of $V$. parahaemolyticus [48]. In this study, the complete genome sequence analysis revealed a mannose-sensitive hemagglutinin gene cluster (mshAC$D E F G I J K L M N$ ) that was required for TFP formation in
$V$. parahaemolyticus $\mathrm{CHN} 25$. Interestingly, the msh gene cluster was significantly down-regulated in the $\triangle$ VpacspA mutant, which indicated a positive regulation of TFP by VpaCspA. The enhanced biofilm formation likely increased the persistence of $V$. parahaemolyticus in the aquatic environment by enhancing low-temperature colonisation of environmental surfaces [49].

Additionally, our transcriptome data also revealed several other molecular mechanisms that facilitated the lowtemperature survival of $V$. parahaemolyticus $\mathrm{CHN} 25$ (Fig. 4). For example, $V p a C s p D$ negatively regulated arginine and proline metabolism, which likely resulted in increased cellular protein flexibility and stability so that efficient functionality could be maintained at the low temperature.

\section{Conclusions}

This study is the first to describe the complete 5,443,401-bp genome sequence $(45.2 \% \mathrm{G}+\mathrm{C})$ of $V$. parahaemolyticus CHN25 (serotype: O5:KUT), which consists of two circular chromosomes and three plasmids with 4,724 predicted protein-encoding genes. One dual-gene and two singlegene deletion mutants of the main CSPs, $V p a C s p A$ and VpaCspD, in $V$. parahaemolyticus CHN25 were successfully constructed. Our data demonstrated that $V p a C s p A$ was a primary CSP in the bacterium, whereas $V p a C s p D$ functioned as a growth inhibitor at $10{ }^{\circ} \mathrm{C}$. Moreover, $V$ pacspD gene expression was negatively regulated by $V p a C s p A$. A global-level transcriptomic analysis revealed distinct gene expression profiles among the three mutants. Approximately $12.4 \%$ of the expressed genes in V. parahaemolyticus CHN25 were significantly altered in the $\triangle V p a c$ $s p A$ mutant at $10{ }^{\circ} \mathrm{C}$, including those involved in amino acid degradation, $\mathrm{ABC}$ transporters, secretion systems, sulphur metabolism and glycerophospholipid metabolism. The low temperature elicited significant changes in expression of $10.0 \%$ of the genes from the $\Delta V p a c s p D$ mutant, including genes that were involved in the phosphotransferase system and in nitrogen and amino acid metabolism. The following major altered metabolic pathways in the $\triangle$ Vpacsp $A D$ mutant radically differed from those in the single-gene mutants at $10^{\circ} \mathrm{C}$ : TCA; PTS; butanoate metabolism; fructose and mannose metabolism; pyruvate, cysteine and methionine metabolism; arginine and proline metabolism; alanine, aspartate and glutamate metabolism; and oxidative phosphorylation. The transcriptome profile comparisons further revealed numerous DEGs that were shared among the three mutants and DERs that were specifically, coordinately and or antagonistically mediated by $V p a C s p A$ and $V p a C s p D$ at a low temperature. $V$. parahaemolyticus appears to have evolved several molecular strategies with a complex gene regulation network for coping with coldinduced damage. The results from this study improve our 
understanding of the genetic basis for low-temperature survival of the most common seafood-borne pathogens worldwide.

\section{Methods}

Bacterial strains, plasmids and culture conditions

Escherichia coli DH5 $\alpha$ Apir (BEINUO Biotech (Shanghai) CO., LD. Shanghai, China) was used as a host strain for DNA cloning. The pDS132 plasmid [50] (a kind gift from Professor Dominique Schneider) was used as a suicide vector to construct the gene deletion mutants. E. coli $\beta 2155$ גpir [51] (a kind gift from Professor Weicheng Bei) was used as a donor strain in the conjugation experiments. The pMMB207 plasmid [52] (Biovector Science Lab, Inc., Beijing, China) was used as a expression vector to construct the reverse mutants. V. parahaemolyticus CHN25 was isolated and characterised by Song et al. [15], Sun et al. [16] and $\mathrm{He}$ et al. [17] and modified for mutant construction by Sun et al. (unpublished). The bacterium was positive for the $t$ th gene but contained no toxic $t d h$ and trh genes [15]. The E. coli strains were routinely incubated in Luria-Bertani (LB) medium (1\% NaCl, $\mathrm{pH} 7.2)$ [53] at $37{ }^{\circ} \mathrm{C}$, and the $V$. parahaemolyticus strains were grown in LB medium ( $3 \% \mathrm{NaCl}, \mathrm{pH}$ 8.5). The diaminopimelic acid (DAP) auxotrophic E. coli strains were grown in LB medium that contained $0.3 \mathrm{mM}$ DAP (Sigma-Aldrich, MO, USA). The medium was supplemented as needed with chloramphenicol to a final concentration of $30 \mu \mathrm{g} / \mathrm{mL}$ for $E$. coli and $5 \mu \mathrm{g} / \mathrm{mL}$ for $V$. parahaemolyticus. Growth curves were determined as previously described [16].

\section{Genome sequencing, assembly, gene functional annotation, and comparative genome analysis}

Whole-genome sequencing of $V$. parahaemolyticus CHN25 was performed at the Chinese National Human Genome Centre (Shanghai, China) using the Genome Sequencer FLX (GS-FLX) system (Roche, Mannheim, Germany), which yielded 177,497 reads with a genome sequencing depth of 22-fold. The sequencing reads were assembled using the Newbler V2.3 software [54]. Gap closure was performed by primer walking and combinatorial PCR as previously described [55]. The final genome assembly was performed using the Phred-Phrap-Consed software packages [56]. Protein-coding genes were predicted using the EasyGene software [57], and functional assignments were inferred based on standalone Basic Local Alignment Search Tool (BLAST) (http://www.ncbi.nlm.nih.gov/BLAST) searches against the SWISS-PROT, GenBank, Clusters of Orthologous Groups of proteins (COGs) [58], and Pfam databases [59]. The rRNA genes were annotated using the FgenesB tool (http://softberry.com/), and tRNA genes were detected using the tRNAscan-
SE programme [60]. IS elements were identified using the IS Finder [VC41]. Prophage-associated genes were predicted using Prophage finder (http://phast.wishartlab.com/). The clustered regularly interspaced short palindromic repeats (CRISPRs) were identified using the CRISPRFinder [61]. Potential virulence factors were detected using the Virulence Factor database (http://www.mgc.ac.cn/VFs/). Whole genome sequence alignments were performed using MUMmer3.2.3 software (http://www.tigr.org/software/mummer/) [62].

\section{Deletion of the VpacspA and VpacspD genes in V. parahaemolyticus CHN25}

Genomic DNA was prepared using the Biospin Bacteria DNA Extraction Kit (BIOER Technology, Hangzhou, China). Plasmid DNA was isolated using the TaKaRa MiniBEST Plasmid Purification Kit Version 3.0 (Japan TaKaRa BIO, Dalian Company, China). A markerless deletion mutant of the VpacspA gene was constructed by homologous recombination (Philippe et al. 2004). Based on the VpacspA gene sequence (213 bp, assigned to VpaChn25A_0413) of the $V$. parahaemolyticus CHN25 genome, primer pairs were designed ( $\operatorname{sp} A$-up-F/cspA-up$\mathrm{R}$ and $\operatorname{csp} A$-down-F/cspA-down- $\mathrm{R}$ ) to target the upstream (528 bp) and downstream (513 bp) sequences, respectively, of the VpacspA gene (see Additional file 4: Table S2). The amplified PCR products were individually digested with corresponding restriction endonucleases (TaKaRa), purified, and ligated into the pDS132 XbaI and SacI cloning sites as previously described $[50,63]$. The ligated DNA was transformed into E. coli DH5 $\alpha$ pir competent cells using the heat-shock method [52]. Positive transformants were screened by colony PCR. The recombinant plasmid, pDS132 $+\triangle$ VpacspA, was subsequently prepared and transformed into DAP auxotroph $E$. coli $\beta 2155$ competent cells. Plate mating assays were performed using $E$. coli $\beta 2155$ (pDS132 $+\Delta$ VpacspA) as the donor and modified $V$. parahaemolyticus $\mathrm{CHN} 25$ as the recipient, as previously described $[15,50]$. Mating was performed at $37{ }^{\circ} \mathrm{C}$ for $12 \mathrm{~h}$ on LB plates $(1.5 \% \mathrm{NaCl}, \mathrm{pH} 7.2)$ that contained $0.3 \mathrm{mM}$ DAP. Cells that were grown on the mating plates were transferred onto LB plates $(3 \% \mathrm{NaCl}, \mathrm{pH} 8.5)$ that contained $5 \mu \mathrm{g} / \mathrm{mL}$ chloramphenicol, which enabled the optimal growth of $V$. parahaemolyticus CHN25. Transconjugants were then inoculated into LB broth $(3 \% \mathrm{NaCl}, \mathrm{pH} 8.5)$ without chloramphenicol and incubated overnight; serial dilutions were spread onto the selective LB agar plates, which were supplemented with $10 \%$ (wt/vol) sucrose. Exconjugants with successful double crossover deletions of the VpacspA gene were screened by colony PCR using the $\operatorname{csp} A$-up-exF and $\operatorname{csp} A$ down-exR primer pair and confirmed by DNA sequencing. The 219-bp VpacspD gene (VpaChn25_1036) deletion was carried out using the method described 
above with the primer designs listed in Additional file 4: Table S2. Furthermore, the VpacspD gene was also deleted from the $\triangle V p a c s p A$ mutant to create the dual-gene deleted $\triangle V p a c s p A D$ mutant.

\section{Construction of the reverse mutants of the VpacspA and VpacspD genes in V. parahaemolyticus CHN25}

The VpacspA gene was amplified from the genomic DNA of $V$. parahaemolyticus CHN25 by PCR with the $\operatorname{csp} A$-com-F and -R primers (Additional file 4: Table S2). The PCR product was, digested with corresponding restriction endonucleases (TaKaRa), purified, and ligated into the expression vector pMMB207 at the SacI and $X b a \mathrm{I}$ cloning sites. The ligated DNA was transformed into E. coli $\mathrm{DH} 5 \alpha$ and positive transformants were screened as described above. The recombinant plasmid pMMB207 + VpacspA was then prepared and transformed into the $\triangle V p a c s p A$ mutant by electrotransformation. The competent cells of the $\triangle V p a c s p A$ mutant was prepared according to the method Hamashima et al. [64] with minor modification. Briefly, the $\triangle V p a c s p A$ mutant was inoculated into $5 \mathrm{~mL}$ Mueller-Hinton Broth (MHB, 3\% NaCl, pH7.0) (Beijing Land Bridge Technology Co., Beijing, China) and incubated at $37{ }^{\circ} \mathrm{C}$. The overnight culture was then collected by centrifugation at 2,700 $\mathrm{g}$ for $4 \mathrm{~min}, 4^{\circ} \mathrm{C}$, and the cell pellet was suspended and washed with cooled EP buffer $(272 \mathrm{mM}$ sucrose, $1 \mathrm{mM} \mathrm{MgCl} 2,7 \mathrm{mM} \mathrm{KH} \mathrm{PO}_{4}-\mathrm{Na}_{2} \mathrm{HPO}_{4}, \mathrm{pH}$ 7.4) for three times. The washed cells were finally suspended with $8 \mathrm{~mL}$ cooled EP buffer, and $200-\mu \mathrm{L}$ aliquots of the cells were stored at $-80{ }^{\circ} \mathrm{C}$. The electrotransformation was performed according to the method [64]. Briefly, $1 \mu \mathrm{g}$ DNA of the plasmid pMMB207+VpacspA was added into $200 \mu \mathrm{L}$ competent cells of the $\Delta$ Vpacsp $A$ mutant, and incubated on ice for $15 \mathrm{~min}$. The electrotransformation was performed at $25 \mathrm{~ms}, 1.5 \mathrm{kV}, 100 \Omega, 25 \mu \mathrm{F}$ conditions using the Gene Pluser XCell (Bio-Rad,USA). Subsequently, $500 \mu \mathrm{L}$ prewarmed $\mathrm{MHB}(3 \% \mathrm{NaCl}, \mathrm{pH} 7.0)$ was quickly added into the electrotransformation mixture, and incubated at $37^{\circ} \mathrm{C}$ for $1 \mathrm{~h}$. The cell culture was then spread onto MHB agar plates supplemented with $5 \mu \mathrm{g} / \mathrm{mL}$ chloramphenicol, and cultured at $37^{\circ} \mathrm{C}$ overnight. The positive electrotransformant ( $\triangle$ VpacspA-com mutant) were screened by colony PCR with primers $\operatorname{csp} A$-com-FR and tlh-FR, and confirmed by DNA sequencing analysis. Similarly, the reverse mutant $\triangle V$ pacspD-com was also constructed with the $\operatorname{csp} D$-com-F and -R primers (Table S2) using the same methods described above.

\section{Illumina RNA sequencing}

Bacterial cells were cultured at $10{ }^{\circ} \mathrm{C}$ until they reached their logarithmic growth phase and were collected by centrifugation. Total RNA was prepared using the RNeasy Protect Bacteria Mini Kit (QIAGEN Biotech Co. Ltd.,
Hilden, Germany) and QIAGEN RNeasy Mini Kit (QIAGEN) according to the manufacturer's protocols. The DNA was removed from the samples with the RNase-Free DNase Set (QIAGEN). Three independently prepared RNA samples were used in each Illumina RNA-sequencing experiment. A wild-type strain that was cultured under identical conditions was used as the control.

The sequencing library construction and Illumina sequencing were conducted at Shanghai Biotechnology Co., Ltd. (Shanghai, China) according to the TruSeq ${ }^{\mathrm{TM}}$ RNA Sample Preparation Guide (Illumina, San Diego, CA, USA). The abundant $16 \mathrm{~S}$ and $23 \mathrm{~S}$ rRNA were depleted using the Ribo-Zero rRNA Removal Kit (Epicentre Biotechnologies, Madison, WI, USA). First-strand cDNA was synthesised using SuperScript II Reverse Transcriptase (Invitrogen, Grand Island, NY, USA) with random hexamer primers. AMPure XP Beads (Beckman Coulter, Beverly, MA, USA) were used to isolate doublestranded cDNA that was synthesised with the Second Strand Master Mix (Invitrogen). The cDNA fragments underwent an end-repair process to convert the overhangs into blunt ends. A single "A" nucleotide was added to the 3 ' ends of each blunt fragment to prevent them from ligating to one another during the adapter ligation reaction. The adapters (data not shown) with corresponding single " $T$ " nucleotides on their 3' ends were ligated, and PCR reactions were performed to enrich the DNA fragments that contained adapter molecules on both ends. Prepared sequencing libraries were quantified with a $\mathrm{Qubit}^{\mathrm{R}} 2.0$ Fluorometre (Invitrogen) and validated using the Agilent High-Sensitivity DNA assay on the Agilent Bioanalyser 2100 system (Agilent Technologies, Santa Clara, CA, USA). Clustering of the index-coded samples was performed on a cBot Cluster Generation System using the TruSeq PE Cluster Kit v3-cBot-HS (Illumina). Sequencing was conducted using an Illumina HiSeq 2500 platform, which generated $2 \times 100$-bp paired-end reads. High quality reads that passed the Illumina quality filters were used for sequence analyses.

\section{Data analysis}

Quality filtration of raw RNA-seq data were performed using the FASTX-Toolkit version 0.0.13 (http://hanonlab.cshl.edu/fastx_toolkit/index.html) to remove the sequencing adapters, identical and low-quality reads, and ribosomal RNA sequences. The resulting clean reads were aligned to the $V$. parahaemolyticus CHN25 genome using the Bowtie2 version 2.0.5 software (http:// bowtie-bio.sourceforge.net/bowtie2/index.shtml). Gene transcriptional abundance of assembling transcripts was estimated according to the reads per kilobase of exon model per million mapped reads (RPKM) method described by Mortazavi et al. [65]. The fold-change was 
determined for each gene by calculating the ratio of the RPKM values between the sample and the control. The genes with criteria fold-changes $\geq 2.0$ or $\leq 0.5$ and $p$-values $<0.05$ relative to the control were defined as DEGs. These DEGs were used for GSEA against the KEGG database (http://www.genome.jp/kegg/), and significantly changed metabolic pathways were identified when the enrichment test p-value fell below 0.05, which was validated by eBioservice (http://sas.ebioservice.com/portal/root/molnet_shbh/index.jsp) (Shanghai Biotechnology Co., Ltd., Shanghai, China) [16].

\section{Real-time reverse transcription PCR}

Selected DEGs and significantly enriched genes in the transcriptome-sequencing analysis were validated by qRTPCR. Oligonucleotide primers were designed using the Primer 5.0 software (http://www. premierbiosoft.com/) (see Additional file 5: Table S3) and synthesised by Shanghai Sangon Biological Engineering Technology Services Co., Ltd. (Shanghai, China). The conditions that were utilised to grow the cells for the qRT-PCR analysis were identical to those used for Illumina RNA sequencing. The qRT-PCR reactions were performed as previously described [16]. Primer specification was confirmed by agarose gel electrophoresis and melting curve analyses, and qRTPCR amplification efficiencies (E) were analysed using the Applied Biosystems 7500 software programme (Applied Biosystems, Foster City, CA, USA). The relative expression ratio $(R)$ of the target gene was calculated based on $\mathrm{E}$ and the crossing point (CP) deviation of the sample versus the control, and it was expressed relative to the reference gene using the delta-delta threshold cycle $\left(\mathrm{C}_{\mathrm{T}}\right)$ method as previously described by Pfaffl [66]. The 16S rRNA gene was used as the reference gene, as previously described [67]. All determinants were performed in triplicate.

\section{Additional files}

Additional file 1: Figure S1. Circular maps of the $V$. parahaemolyticus CHN25 chromosomes. (a) and (b) represent the larger and smaller chromosomes of $V$. parahaemolyticus CHN25, respectively. Each circle in the grey lines, except for the two innermost circles, illustrates specific features on the plus (outer region) and minus (inner region) strands. The lines and boxes in the three outermost circles are coloured according to the COG categories. The circles indicate the following from the outside inwards: first circle, predicted protein-coding genes; second circle, classified essential genes, including cell division, replication, transcription, translation, and amino acid metabolism; third circle, tRNA genes and rRNA operons; fourth circle, GC-skew (values above zero are red, values below zero are blue); fifth circle, GC content. (TIF 22070 kb)

Additional file 2: Figure S2. Circular maps of the $V$. parahaemolyticus CHN25 plasmids. (a)-(c): each circle in the grey lines, except for the two innermost circles, illustrates specific features on the plus (outer region) and minus (inner region) strands. Lines and boxes in the three outermost circles are coloured according to the COG categories. The circles indicate the following from the outside inwards: first circle, predicted protein-coding genes; second circle, GC-skew (values above zero in red, values below zero in blue); and third circle, GC content. (TIF $15096 \mathrm{~kb}$ )

Additional file 3: Table S1. The DERs in the $\triangle V p a c s p A, \triangle V p a c s p D$ and $\triangle V p a c s p A D$ mutants of $V$. parahaemolyticus $C H N 25$ at low temperatures. (DOC $96 \mathrm{~kb}$ )

Additional file 4: Table S2. Oligonucleotide primers for mutant construction used in this study. (DOC $48 \mathrm{~kb}$ )

Additional file 5: Table S3. Oligonucleotide primers used for the RT-PCR analysis in this study. (DOC $59 \mathrm{~kb}$ )

\section{Abbreviations}

ABC: ATP-binding cassette; BCCTs: Betaine-choline-carnitine transporters; BLAST: basic local alignment search tool; CAMP: 3'-5'-cyclic adenosine monophosphate; COGs: clusters of orthologous groups; CP: crossing point; CRISPRs: clustered regularly interspaced short palindromic repeats; CRP: CAMP receptor protein; CSPs: Cold shock proteins; DAP: Diaminopimelic acid; DEGs: Differentially expressed genes; DHAP: Dihydroxyacetone phosphate; GB: Glycine betaine; G3P: Sn-glycerol-3-phosphate; GSEA: Gene set enrichment analysis; ISs: Insertion sequences; R-M: Restriction and modification; KEGG: Kyoto encyclopaedia of genes and genomes; PTS: Phosphotransferase system; TCA: Tricarboxylic acid cycle; TFP: Type IV pili; TSS: Secretion system types

\section{Acknowledgements}

We acknowledge Professor Dominique Schneider for kindly providing us the plasmid pDS132 for the construction of three gene deletion mutants.

\section{Funding}

The work was supported by a Grant No.09320503600 from Shanghai Municipal Science and Technology Commission, Grants No.B-9500-10-0004, No.13YZ098, and No.ZZhy12028 from Shanghai Municipal Education Commission, and Grants No.31271830 and No.31671946 from National Natural Science Foundation of China. The funding agencies had no role in the design of the study and collection, analysis, and interpretation of data and in writing the manuscript.

\section{Availability of data and materials}

The annotated complete genome sequence of $V$. parahaemolyticus CHN25 has been deposited in the GenBank database under the accession numbers: C25C1.sqn C25C1 CP010883, C25C2.sqn C25C2 CP010884, C25P1.sqn C25P1 CP010885, C25P2.sqn C25P2 CP010886 and C25P3.sqn C25P3 CP010887. The RNA-Seq data have been deposited in the NCBI Gene Expression Omnibus database (http://www.ncbi.nlm.nih.gov/geo/) under the accession number GSE65998.

\section{Authors' contributions}

$C Z, B S, T L, H Z, F S, M Y, W B, X P, Q S, L X$ and LC participated in the design and or discussion of the study. BS performed genome gap closure, gene annotation and comparative genome analysis. $\mathrm{HZ}$ directed the sequencing and comparative genome analysis. WG and WH assisted the genome sequencing and gene annotation. CZ carried out the major experiments in gene deletion mutant and transcriptomic analysis. YW constructed gene reverse mutants and analyzed their growth curves. CZ, BS, TL and LC analyzed the data. LC wrote the manuscript, and $\mathrm{HZ}, \mathrm{QS}$ and $\mathrm{LX}$ revised it for important improvement. All authors read and approved the final manuscript.

\section{Consent for publication}

Not applicable.

\section{Competing interests}

The authors declare no conflict of interest.

Ethics approval and consent to participate Not applicable.

\section{Publisher's Note}

Springer Nature remains neutral with regard to jurisdictional claims in published maps and institutional affiliations. 


\section{Author details}

'Key Laboratory of Quality and Safety Risk Assessment for Aquatic Products on Storage and Preservation (Shanghai), China Ministry of Agriculture; College of Food Science and Technology, Shanghai Ocean University, 999 Hu Cheng Huan Road, Shanghai 201306, People's Republic of China. ${ }^{2}$ College of Information Technology, Shanghai Ocean University, $999 \mathrm{Hu}$ Cheng Huan Road, Shanghai 201306, People's Republic of China. ${ }^{3}$ Shanghai-MOST Key Laboratory of Disease and Health Genomics, Chinese National Human Genome Centre at Shanghai, Shanghai 201203, People's Republic of China. ${ }^{4}$ Shanghai Hanyu Bio-lab, 151 Ke Yuan Road, Shanghai 201203, People's Republic of China. ${ }^{5}$ Shanghai Institute for Food and Drug Control, 1500 Zhang Heng Road, Shanghai 201203, People's Republic of China. ${ }^{6}$ State Key Laboratory of Agricultural Microbiology, Laboratory of Animal Infectious Diseases, College of Animal Science \& Veterinary Medicine, Huazhong Agricultural University, Wuhan, Hubei 430070, People's Republic of China. ${ }^{7}$ Archaea Centre, Department of Biology, University of Copenhagen, Ole Maaløes Vej 5, DK2200, Copenhagen N, Denmark. ${ }^{8}$ Shanghai Center for Bioinformation Technology, 1278 Keyuan Road, Shanghai 201203, People's Republic of China.

\section{Received: 9 August 2016 Accepted: 10 May 2017}

\section{Published online: 05 June 2017}

\section{References}

1. Ceccarelli D, Hasan NA, Huq A, Colwell RR. Distribution and dynamics of epidemic and pandemic Vibrio parahaemolyticus virulence factors. Front Cell Infect Microbiol. 2013;3:97.

2. Su YC, Liu C. Vibrio parahaemolyticus: a concern of seafood safety. Food Microbiol. 2007;24:549-58.

3. Fujino T, Okuno Y, Nakada D, Aoyama A, Fukai K, Mukai T, et al. On the bacteriological examination of shirasu food poisoning. Med J Osaka Univ. 1953:4:299-304.

4. Makino K, Oshima K, Kurokawa K, Yokoyama K, Uda T, Tagomori K, et al. Genome sequence of Vibrio parahaemolyticus: a pathogenic mechanism distinct from that of $V$. cholerae. Lancet. 2003:361 (9359):743-9.

5. Jensen RV, Depasquale SM, Harbolick EA, Hong T, Kernell AL, Kruchko DH, et al. Complete genome sequence of prepandemic Vibrio parahaemolyticus BB22OP. Genome Announc. 2013;1 (1):e00002-12.

6. Kalburge SS, Polson SW, Boyd Crotty K, Katz L, Turnsek M, Tarr CL, et al. Complete genome sequence of Vibrio parahaemolyticus environmental strain UCM-V493. Genome Announc. 2014;2(2):e00159-14.

7. Gonzalez-Escalona N, Strain EA, De Jesus AJ, Jones JL, Depaola A. Genome sequence of the clinical 04:K12 serotype Vibrio parahaemolyticus strain 10329. J Bacteriol. 2011:193:3405-6.

8. Yang YT, Chen IT, Lee CT, Chen CY, Lin SS, Hor LI, et al. Draft genome sequences of four strains of Vibrio parahaemolyticus, three of which cause early mortality syndrome/acute hepatopancreatic necrosis disease in shrimp in China and Thailand. Genome Announc. 2014;2(5):e00816-14.

9. Haendiges J, Timme R, Allard M, Myers RA, Payne J, Brown EW, et al. Draft genome sequences of clinical Vibrio parahaemolyticus strains isolated in Maryland (2010 to 2013). Genome Announc. 2014;2(4):e00776-14.

10. Horn G, Hofweber R, Kremer W, Kalbitzer HR. Structure and function of bacterial cold shock proteins. Cell Mol Life Sci. 2007;64:1457-70.

11. Wouters JA, Rombouts FM, Kuipers OP, de Vos WM, Abee T. The role of cold-shock proteins in low-temperature adaptation of food-related bacteria. Syst Appl Microbiol. 2000;23:165-73.

12. Yamanaka K, Zheng W, Crooke E, Wang YH, Inouye M. CspD, a novel DNA replication inhibitor induced during the stationary phase in Escherichia coli. Mol Microbiol. 2001;39:1572-84.

13. Kim Y, Wang $X$, Zhang XS, Grigoriu S, Page R, Peti W, et al. Escherichia coli toxin/antitoxin pair MqsR/MqsA regulate toxin CspD. Environ Microbiol. 2010;12:1105-21.

14. Yang L, Zhou D, Liu X, Han H, Zhan L, Guo Z, et al. Cold-induced gene expression profiles of Vibrio parahaemolyticus: a time-course analysis. FEMS Microbiol Lett. 2009:291:50-8.

15. Song $Y$, Y U P, Li B, Pan Y, Zhang X, Cong J, et al. The mosaic accessory gene structures of the SXT/R391-like integrative and conjugative elements derived from Vibrio spp. isolated from aquatic products and environment in the yangtze river estuary, China. BMC Microbiol. 2013; 13:214.
16. Sun $X$, Liu T, Peng $X$, Chen L. Insights into Vibrio parahaemolyticus CHN25 response to artificial gastric fluid stress by transcriptomic analysis. Int J Mol Sci. 2014;15:22539-62.

17. Yu He,Hua Wang, Lanming Chen. Comparative secretomics analysis reveals novel virulence-associated factors of Vibrio parahaemolyticus. Front Microbiol. 2015;6:707.

18. Xue $H, X u Y$, Boucher $Y$, Polz MF. High frequency of a novel filamentous phage, VCY phi, within an environmental Vibrio cholerae population. Appl Environ Microbiol. 2012;78:28-33.

19. Fall S, Mercier A, Bertolla F, Calteau A, Gueguen L, Perriere G, et al. Horizontal gene transfer regulation in bacteria as a "spandrel" of DNA repair mechanisms. PLoS One. 2007;2:e1055.

20. Ma L, Zhang Y, Yan X, Guo L, Wang L, Qiu J, et al. Expression of the type VI secretion system 1 component Hcp1 is indirectly repressed by OpaR in Vibrio parahaemolyticus. Sci World J. 2012;2012:982140.

21. Bakermans C, Tollaksen SL, Giometti CS, Wilkerson C, Tiedje JM, Thomashow MF. Proteomic analysis of Psychrobacter cryohalolentis K5 during growth at subzero temperatures. Extremophiles. 2007;11:343-54.

22. Ishikawa T, Sabharwal D, Broms J, Milton DL, Sjostedt A, Uhlin BE, et al. Pathoadaptive conditional regulation of the type VI secretion system in Vibrio cholerae 01 strains. Infect Immun. 2012;80:575-84.

23. Ayala-del-Rio HL, Chain PS, Grzymski JJ, Ponder MA, Ivanova N, Bergholz PW, et al. The genome sequence of Psychrobacter arcticus 273-4, a psychroactive Siberian permafrost bacterium, reveals mechanisms for adaptation to low-temperature growth. Appl Environ Microbiol. 2010;76:2304-12.

24. Adekoya OA, Helland R, Willassen NP, Sylte I. Comparative sequence and structure analysis reveal features of cold adaptation of an enzyme in the thermolysin family. Proteins. 2006;62:435-49.

25. Thorvaldsen S, Hjerde E, Fenton C, Willassen NP. Molecular characterization of cold adaptation based on ortholog protein sequences from Vibrionaceae species. Extremophiles. 2007;11:719-32.

26. Motin VL, Georgescu AM, Fitch JP, Gu PP, Nelson DO, Mabery SL, et al. Temporal global changes in gene expression during temperature transition in Yersinia pestis. J Bacteriol. 2004;186:6298-305.

27. Postma PW, Lengeler JW, Jacobson GR. Phosphoenolpyruvate: carbohydrate phosphotransferase systems of bacteria. Microbiol Rev. 1993;57:543-94.

28. Fraser KR, Tuite NL, Bhagwat A, O'Byrne CP. Global effects of homocysteine on transcription in Escherichia coll: induction of the gene for the major cold-shock protein, CspA. Microbiol. 2006;152(Pt 8):2221-31.

29. Bakhlanova IV, Dudkina AV, Baitin DM. Enzymatic control of homologous recombination in Escherichia coli cells and hyper-recombination. Mol Biol (Mosk). 2013;47:205-17.

30. Rkenes TP, Lamark T, Strom AR. DNA-binding properties of the Betl repressor protein of Escherichia coli: the inducer choline stimulates Betl-DNA complex formation. J Bacteriol. 1996;178:1663-70.

31. Ongagna-Yhombi SY, McDonald ND, Boyd EF. Deciphering the role of multiple Betaine-Carnitine-Choline transporters in the halophile Vibrio parahaemolyticus. Appl Environ Microbiol. 2015;81:351-63.

32. Cayley S, Record Jr MT. Roles of cytoplasmic osmolytes, water, and crowding in the response of Escherichia coli to osmotic stress: biophysical basis of osmoprotection by glycine betaine. Biochemistry. 2003;42:12596-609.

33. Hoffmann T, Bremer E. Protection of Bacillus subtilis against cold stress via compatible-solute acquisition. J Bacteriol. 2011;193:1552-62.

34. Kaleta C, Gohler A, Schuster S, Jahreis K, Guthke R, Nikolajewa S. Integrative inference of gene-regulatory networks in Escherichia coli using information theoretic concepts and sequence analysis. BMC Syst Biol. 2010;4:116.

35. Humbert MV, Rasia RM, Checa SK, Soncini FC. Protein signatures that promote operator selectivity among paralog MerR monovalent metal ion regulators. J Biol Chem. 2013;288:20510-9.

36. Noor R, Murata M, Nagamitsu H, Klein G, Raina S, Yamada M. Dissection of sigma(E)-dependent cell lysis in Escherichia coli: roles of RpoE regulators RseA, RseB and periplasmic folding catalyst PpiD. Genes Cells. 2009;14:885-99.

37. Schwoppe C, Winkler HH, Neuhaus HE. Connection of transport and sensing by UhpC, the sensor for external glucose-6-phosphate in Escherichia coli. Eur J Biochem. 2003;270:1450-7.

38. Bresolin G, Neuhaus K, Scherer S, Fuchs TM. Transcriptional analysis of long-term adaptation of Yersinia enterocolitica to low-temperature growth. J Bacteriol. 2006;188:2945-58.

39. Dahlsten E, Zhang Z, Somervuo P, Minton NP, Lindstrom M, Korkeala H. The cold-induced two-component system CBO0366/CBO0365 regulates 
metabolic pathways with novel roles in group I Clostridium botulinum ATCC 3502 cold tolerance. Appl Environ Microbiol. 2014;80:306-19.

40. Budde I, Steil L, Scharf C, Volker U, Bremer E. Adaptation of Bacillus subtilis to growth at low temperature: a combined transcriptomic and proteomic appraisal. Microbiol. 2006;152(Pt 3):831-53.

41. Chan YC, Hu Y, Chaturongakul S, Files KD, Bowen BM, Boor KJ, et al. Contributions of two-component regulatory systems, alternative sigma factors, and negative regulators to Listeria monocytogenes cold adaptation and cold growth. J Food Prot. 2008;71:420-5.

42. Hunke S, Keller R, Muller VS. Signal integration by the Cpx-envelope stress system. FEMS Microbiol Lett. 2012;326:12-22.

43. Vogt SL, Nevesinjac AZ, Humphries RM, Donnenberg MS, Armstrong GD, Raivio TL. The Cpx envelope stress response both facilitates and inhibits elaboration of the enteropathogenic Escherichia coli bundle-forming pilus. Mol Microbiol. 2010;76:1095-110.

44. Heller KB, Lin EC, Wilson TH. Substrate specificity and transport properties of the glycerol facilitator of Escherichia coli. J Bacteriol. 1980;144:274-8.

45. Domka J, Lee J, Bansal T, Wood TK. Temporal gene-expression in Escherichia coli K-12 biofilms. Environ Microbiol. 2007;9:332-46.

46. Lee JJ, Lee G, Shin JH. sigma(B) affects biofilm formation under the dual stress conditions imposed by adding salt and low temperature in Listeria monocytogenes. J Microbiol. 2014;52:849-55.

47. Kandror O, DeLeon A, Goldberg AL. Trehalose synthesis is induced upon exposure of Escherichia coli to cold and is essential for viability at low temperatures. Proc Natl Acad Sci U S A. 2002;99:9727-32.

48. Shime-Hattori A, lida T, Arita M, Park KS, Kodama T, Honda T. Two type IV pili of Vibrio parahaemolyticus play different roles in biofilm formation. FEMS Microbiol Lett. 2006;264:89-97.

49. Stauder M, Vezzulli L, Pezzati E, Repetto B, Pruzzo C. Temperature affects Vibrio cholerae $\mathrm{O} 1 \mathrm{El}$ Tor persistence in the aquatic environment via an enhanced expression of GbpA and MSHA adhesins. Environ Microbiol Rep. 2010;2:140-4.

50. Philippe N, Alcaraz JP, Coursange E, Geiselmann J, Schneider D. Improvement of pCVD442, a suicide plasmid for gene allele exchange in bacteria. Plasmid. 2004;51:246-55.

51. Dehio C, Meyer M. Maintenance of broad-host-range incompatibility group P and group Q plasmids and transposition of Tn5 in Bartonella henselae following conjugal plasmid transfer from Escherichia coli. J Bacteriol. 1997;179:538-40

52. Morales VM, Bäckman A, Bagdasarian M. A series of wide-host-range low-copy-number vectors that allow direct screening for recombinants. Gene. 1991;97:39-47.

53. Sambrook J, Russell DW, Russell DW. Molecular cloning: a laboratory manual. New York: Cold spring harbor laboratory press; 2001.

54. Mundry M, Bornberg-Bauer E, Sammeth M, Feulner PG. Evaluating characteristics of de novo assembly software on 454 transcriptome data: a simulation approach. PLoS One. 2012;7:e31410.

55. Chen L, Brügger K, Skovgaard M, Redder P, She Q, Torarinsson E, et al. The genome of Sulfolobus acidocaldarius, a model organism of the Crenarchaeota. J Bacteriol. 2005;87:4992-9.

56. Gordon D, Abajian C, Green P. Consed: a graphical tool for sequence finishing. Genome Res. 1998;8:195-202.

57. Larsen TS, Krogh A. EasyGene-a prokaryotic gene finder that ranks ORFs by statistical significance. BMC Bioinformat. 2003;4:21.

58. Tatusov RL, Natale DA, Garkavtsev IV, Tatusova TA, Shankavaram UT, Rao BS, et al. The COG database: new developments in phylogenetic classification of proteins from complete genomes. Nucleic Acids Res. 2001;29:22-8

59. Bateman A, Birney E, Cerruti L, Durbin R, Etwiller L, Eddy SR, et al. The Pfam protein families database. Nucleic Acids Res. 2002;30:276-80.

60. Lowe TM, Eddy SR. tRNAscan-SE: a program for improved detection of transfer RNA genes in genomic sequence. Nucleic Acids Res. 1997;25:955-64.

61. Grissa I, Vergnaud G, Pourcel C. CRISPRFinder: a web tool to identify clustered regularly interspaced short palindromic repeats. Nucleic Acids Res. 2007;35:W52-57. Web Server issue.

62. Kurtz S, Phillippy A, Delcher AL, Smoot M, Shumway M, Antonescu C, et al. Versatile and open software for comparing large genomes. Genome Biol. 2004;5:R12.

63. Li Y, Pan Y, She Q, Chen L. A novel carboxyl-terminal protease derived from Paenibacillus lautus CHN26 exhibiting high activities at multiple sites of substrates. BMC Biotechnol. 2013;13:89.
64. Hamashima H, Iwasaki M, Arai T. A simple and rapid method for transformation of Vibrio species by electroporation. Electrop Protoc Microorgan. 1995;47:155-60.

65. Mortazavi A, Williams BA, McCue K, Schaeffer L, Wold B. Mapping and quantifying mammalian transcriptomes by RNA-Seq. Nat Methods. 2008;5:621-8.

66. Pfaffl MW. A new mathematical model for relative quantification in real-time RT-PCR. Nucleic Acids Res. 2001;29:e45.

67. Chen SY, Jane WN, Chen YS, Wong HC. Morphological changes of Vibrio parahaemolyticus under cold and starvation stresses. Int J Food Microbiol. 2009;129:157-65.

\section{Submit your next manuscript to BioMed Central and we will help you at every step:}

- We accept pre-submission inquiries

- Our selector tool helps you to find the most relevant journal

- We provide round the clock customer support

- Convenient online submission

- Thorough peer review

- Inclusion in PubMed and all major indexing services

- Maximum visibility for your research

Submit your manuscript at www.biomedcentral.com/submit
Biomed Central 